\title{
Signatures of Planets in Spatially Unresolved Debris Disks
}

\author{
Amaya Moro-Martín ${ }^{1,2}$, Sebastian Wolf ${ }^{2,3} \&$ Renu Malhotra ${ }^{4}$ \\ amaya@as.arizona.edu, swolf@astro.caltech.edu, renu@lpl.arizona.edu
}

\begin{abstract}
Main sequence stars are commonly surrounded by debris disks, composed of cold dust continuously replenished by a reservoir of undetected dust-producing planetesimals. In a planetary system with a belt of planetesimals (like the Solar System's Kuiper Belt) and one or more interior giant planets, the trapping of dust particles in the mean motion resonances with the planets can create structure in the dust disk, as the particles accumulate at certain semimajor axes. Sufficiently massive planets may also scatter and eject dust particles out of a planetary system, creating a dust depleted region inside the orbit of the planet. In anticipation of future observations of spatially unresolved debris disks with the Spitzer Space Telescope, we are interested in studying how the structure carved by planets affects the shape of the disk's spectral energy distribution (SED), and consequently if the SED can be used to infer the presence of planets. We numerically calculate the equilibrium spatial density distributions and SEDs of dust disks originated by a belt of planetesimals in the presence of interior giant planets in different planetary configurations, and for a representative sample of chemical compositions. The dynamical models are necessary to estimate the enhancement of particles near the mean motion resonances with the planets, and to determine how many particles drift inside the planet's orbit. Based on the SEDs and predicted Spitzer colors we discuss what types of planetary systems can be distinguishable from one another and the main parameter degeneracies in the model SEDs.
\end{abstract}

Subject headings: circumstellar matter — interplanetary medium — Kuiper Belt methods: n-body simulations — planetary systems — radiative transfer

\section{Introduction}

Long before planets were discovered by the Doppler technique in the mid 90's, there was indirect evidence of planet building from observations of debris disks around several main sequence

\footnotetext{
${ }^{1}$ Steward Observatory, University of Arizona, 933 N. Cherry Avenue, Tucson, AZ 85721, USA

${ }^{2}$ Max-Planck-Institut fur Astronomie, Königstuhl 17, 69117 Heidelberg, Germany

${ }^{3}$ Division of Physics, Mathematics, and Astronomy, California Institute of Technology, Pasadena, CA 91125, USA

${ }^{4}$ Department of Planetary Sciences, University of Arizona, 1629 E. University Boulevard, Tucson, AZ 85721, USA
} 
stars (Backman \& Paresce 1993 and reference therein). Stars harboring debris disks are too old to have remnants of the primordial disk from which the star itself once formed. The timescale of dust grain removal due to Poynting-Robertson (P-R) drag for a solar type star is $\mathrm{t}_{P R} \sim 400 \times \mathrm{R}^{2} / \beta$ years $\sim 10^{5}$ years, where $\mathrm{R}$ is the grain distance to the central star in $\mathrm{AU}$, and $\beta$ is the ratio of the radiation pressure force to the gravitational force (in the range 0-0.5). Grain removal due to radiation pressure is much faster, as the particles escape quickly on hyperbolic orbits. Grain removal timescales are therefore much shorter than the age of main sequence stars, $>10^{7}$ years, indicating that these dust disks are not primordial. These Solar System-sized disks of micronsized grains are thought to be the result of mutual collisions between asteroid-like bodies or the evaporation of comets close to the star (Backman \& Paresce 1993).

In the standard scenario, debris disks are generated mainly at early times when planetesimals are forming and colliding frequently; this period would coincide with the heavy bombardment in the early Solar System. In agreement with this scenario, far-infrared surveys with the Infrared Space Observatory $(I S O)$ indicate that disk detection drops abruptly at $~ 0.4$ Gyr (Habing et al. 2001) and that the mass decline in the disks is proportional to $\mathrm{t}^{-2}$ between stellar ages of $10 \mathrm{Myr}$ and 1 Gyr (Spangler et al. 2001). The processes responsible for the clearing of dust are stellar winds, radiation pressure, sublimation, collisions and gravitational scattering by giant planets. However, Greaves \& Wyatt (2003) found recently that a small number of stars with an age of a few Gyr do have disks, in disagreement with the standard scenario. They claim that Habing et al. (2001) and Spangler et al. (2001) results are biased toward younger ages, as they were preferentially detecting A (younger) stars. These new results indicate that debris disk duration is $\sim 0.5$ Gyr, and may occur at any time during the main sequence, and that the disk mass decline in time is slow, not steeper than $\mathrm{t}^{-1 / 2}$. It is suggested that the disks have "on" and "off" stages with large differences in dust mass. Furthermore, recent observations by the Spitzer Space Telescope (Rieke et al. 2005 and Gorlova et al. 2004) suggest that in some cases the debris disk phenomena may be the result of stochastic catastrophic collisional events, rather than a continuous generation of dust for long periods of time.

The observation of debris disks indicates that planetesimal formation is indeed a common by-product of the star formation process. One would expect, therefore, that some stars harbor both giant planets and extended emission, as is the case of the Sun. Submillimeter surveys have been designed to verify this assumption (Greaves et al. 2004), but the results are inconclusive due to the low sensitivity of the observations (down to a dust mass limit of $0.02 \mathrm{M}_{\text {Earth }}$ ), and the youth of some of the stars (with difficult accurate radial velocity measurements). This situation is dramatically changing thanks to the high sensitivity of the Spitzer MIPS instrument. A preview of a large Spitzer/MIPS GTO program confirms that out of 26 FGK field stars known to have planets by radial velocity studies, 6 show $70 \mu \mathrm{m}$ excess at $3-\sigma$ confidence level, implying the presence of cool material $(<100 \mathrm{~K}$ ) located beyond $10 \mathrm{AU}$ (Beichman et al. 2005). These stars, with a median age of $4 \mathrm{Gyr}$, are the first to be identified as having both well-confirmed planetary systems and well-confirmed IR excesses (Beichman et al. 2005). Additionally, there are high-resolution images 
of few debris disks that show the presence of density structure (Wilner et al. 2002 and Greaves et al. 1998). Dynamical models have shown that planets can sculpt the disks, creating gaps, arcs, rings, warps and clumps of dust (Roques et al 1994; Liou \& Zook 1999; Mouillet et al. 1997; Wyatt et al 1999; Moro-Martín \& Malhotra 2002 and Kuchner \& Holman 2003), and therefore, it is suggested that these images confirm that debris disks and long-period planets coexist (Ozernoy et al. 2000; Quillen \& Thorndike 2002 and Wilner et al. 2002).

We all know what is the fundamental question that missions like Terrestrial Planet Finder will try to address: are there other potential sites for Life, far beyond our Earth? The study of debris disks can tell us where to look, and where not to look. The reason why this is the case is the following: debris disk structure is sensitive to the presence of long-period planets, complementing a parameter space not covered by radial velocity and transient surveys, and an understanding of the orbits of long period planets is fundamental for the study of the stability of orbits in the habitable zone, where terrestrial planets could form and survive. In the future, the Atacama Large Millimeter Array (ALMA) will be able to image the dust in debris disks with an order of magnitude higher spatial resolution (10 milliarcseconds) than the Very Large Array and the Hubble Space Telescope, in systems which are more than an order of magnitude fainter; i.e. it will be able to search for analogs of the Kuiper Belt dust disk. Careful modeling of the dust density distributions will be essential to interpret these high spatial resolution observational data in terms of planetary architecture. In the more immediate future, Spitzer will carry out spectrophotometric observations of hundreds of circumstellar disks that will likely be spatially unresolved. This is why we are interested in studying how the dust density structure affects the shape of the disk spectral energy distribution (SED) and consequently if the SED can be used to infer the presence of planets.

A first general approach to the simulation of SEDs of debris disk systems using analytic dust density distributions has been undertaken by Wolf \& Hillenbrand (2003; hereafter WH03). This study made clear that the SED analysis strongly depends on the assumed density distribution, in particular of the smallest grain population. In contrast to the former approach by WH03, however, the dust density distribution of a debris disk should not be chosen a priori because it cannot be defined independently from the SED of the embedded star or the dust grain properties (grain size distribution, density and optical constants). In this study we use a self-consistent combination of existing numerical tools for the simulation of debris disk dust density distributions (that take into account the interplay between the central star SED, the grain properties and the dust dynamics), and the radiative transfer simulations in WH03 for the calculation of their emergent SEDs.

We study hypothetical debris disks originating from a belt of planetesimals [uniformly distributed from 35 to $50 \mathrm{AU}$; similar to the Kuiper Belt (KB)] and evolving under the effect of gravitational perturbation from interior giant planets in various planetary configurations. In MoroMartín \& Malhotra (2002 and 2003) we described how in the Solar System, the trapping of particles in mean motion resonances (MMRs) with the giant planets can create structure in the KB dust disk as the dust particles accumulate at certain semimajor axes. We found that for the Solar System planetary configuration, the azimuthal structure of the dust disk is not predictable in detail 
(with simulations of a small number $\mathrm{N}$ of particles, $\mathrm{N} \sim 100$ ) because it depends sensitively on the times of residence in the various resonances and these are highly variable and unpredictable. After careful analysis we concluded that even though the particle dynamics is chaotic, our method could robustly estimate the equilibrium radial density distribution of dust. We found that the combination of radiation forces and planetary perturbations causes the dust disk to be depleted inward of Saturn's orbit and spread outward beyond the KB source region, and the particle size distribution to flatten.

As a complement to $\mathrm{WH} 03$, in this paper we investigate to what extent these planet-induced changes in the radial spatial density distribution and the particle size distribution affect the dust disk SED, and how these effects might be exploited to infer the presence of giant planets in spatially unresolved debris disks. In $\S 2$, we calculate the spatial density distribution of dust grains of different sizes, corresponding to different $\beta$ values. The dynamical evolution of the dust particle, and therefore the dust density distribution, depend only on the parameter $\beta$, which is a function of the grain size and composition and the stellar spectrum (assumed to be solar). The resulting three-dimensional density distribution is transformed into a one-dimensional radial density distribution, which is sufficient to calculate the disk SED because we assume that the disk is optically thin, and therefore the temperature distribution of the grains depends only on the distance to the central star. In $\S 3$, we select a representative sample of chemical compositions based on debris disk spectroscopic observations, including Fe-rich and Fe-poor silicates (crystalline and amorphous olivine and pyroxene) and carbonaceous materials. For each chemical composition, using laboratory optical constants and Mie theory, we calculate the grain radiation pressure coefficients averaged over the stellar spectrum. This allow us to find the correspondence between $\beta$ and the particle radius. Once the particle size is known for each $\beta$ value and selected chemical composition, in $\S$ 4 we calculate, using the same one-dimensional radiative transfer code as in WH03, the emitted dust SED (plus the stellar scattered light) for each single particle size, single composition disk. We later weight and combine these SEDs in order to consider certain particle size distributions. Because this work is part of the Spitzer FEPS Legacy project, which is focused on the detection and characterization of debris disks around F,G and K nearby stars, we use a solar type star as the central heating source and a distance of $50 \mathrm{pc}$ (the median distance of the FEPS targets). A disk mass of $10^{-10} \mathrm{M}_{\odot}$ has been assumed to ensure that the disk is optically thin to stellar radiation, even along the mid plane, and that mutual grain collisions are not important. We further assume that the disks have little or no gas, so that the dust dynamics is controlled by gravitation and radiation forces only. The modeling does not include mutual grain collisions and gas drag (i.e. the systems under consideration are old optically thin disks); quick sublimation of icy fraction (a rapid mass loss can cause the grain's orbit to become more eccentric); grain erosion due to sputtering by solar wind particles; and Lorentz forces due to interplanetary magnetic fields. For a more detailed description of the applied numerical model and an estimate of the limitations mentioned above we refer to Moro-Martín \& Malhotra (2002 and 2003).

A schematic diagram explaining modeling approach is shown in Figure 1. The goal is to 
investigate how the presence of planets affects the debris disk's SED by comparing systems with planets (of different masses and different semimajor axis) and systems without planets, and to derive and analyze the parameter degeneracies in the model SEDs.

\section{Dust Spatial Density Distributions}

A detailed description of the dynamical models used to calculate the dust spatial density distributions, the numerical algorithm used to integrate the equations of motion, and the uncertainties inherent in the prediction of structure, owing to the chaotic dynamics of dust orbital evolution, were given by Moro-Martín \& Malhotra (2002). Here, we briefly overview the main ideas.

We follow numerically, from source to sink, the evolution of sets of 100 dust particles from an outer belt of planetesimals similar to the KB under the combined effects of solar gravity, solar radiation pressure, Poynting-Robertson (P-R) and solar wind drag, and the gravitational forces of the planets. We study the following planetary configurations:

1. The Solar System with 7 planets (excluding Mercury and Pluto). The parent bodies of the dust particles are assumed to be uniformly distributed in orbits with semimajor axis between 35 and $50 \mathrm{AU}$, eccentricities such that the periastron distances are between 35 and $50 \mathrm{AU}$ (i.e. between 0 and 0.3 ), and inclinations between $0^{\circ}$ and $17^{\circ}$, in approximate accord with current estimates of the orbital distribution of KB objects (KBOs; Malhotra et al. 2000).

2. A system with the same distribution of parent bodies as above but without planets.

3. Nine single-planet systems with a planet mass of 1,3 and $10 \mathrm{M}_{J u p}$ in a circular orbit with semimajor axis of 1,5 , and $30 \mathrm{AU}$. The parent bodies are distributed in orbits with semimajor axis between 35 and $50 \mathrm{AU}$, eccentricities between 0 and 0.05 and inclinations between 0 and $3^{\circ}$, to account for the fact that a thinner planetesimal disk may be more realistic when a single giant planet is present. [In this study we found that the difference between the "thick" and the "thin" planetesimal disks is negligible]. For the models with a single planet at $30 \mathrm{AU}$, we ignored the dust particles originated from the 30 planetesimals that lie between 35-40 AU [i.e. inside the 3:2 MMR]. This is because due to multiple close encounters with the planet, we do not expect to have planetesimals in stable orbits in that region.

4. A system with the same distribution of parent bodies as above but without planets.

For the first two cases (KB-like disk with and without Solar System planets), we run 17 sets of 100 particles each, corresponding to 17 different particle sizes, with $\beta$ values ranging from 0.00156

to 0.4 , distributed to get a uniform logarithmic sampling in particle size (see Figure 5). For the rest of the systems (i.e. the "thinner" planetesimal disk with and without single planets), we run a subset of nine $\beta$ values, also ranging from 0.00156 to 0.4 . 
We assume that the dust is generated from a constant grinding down of planetesimals due to mutual collisions or collisions with interstellar grains, adopting a constant dust production rate throughout the planetesimal belt (between $35 \mathrm{AU}$ and $50 \mathrm{AU}$ ). The sinks of dust included in our numerical simulations are ejection into unbound orbits, accretion onto the planets, and orbital decay to less than $\mathrm{r}_{\text {min }}$, where $\mathrm{r}_{\text {min }}=0.5 \mathrm{AU}$ (astrocentric distance) for all models, except for those where the single planet is at $1 \mathrm{AU}$, for which we use $\mathrm{r}_{\text {min }}=0.1 \mathrm{AU}$ instead. Assuming that the dust production rate is in equilibrium with the loss rate, and the dust particle dynamics is ergodic (i.e. the time-weighting reflects the spatial density), we can obtain equilibrium density distributions by recording the positions of these 100 particles at equal time intervals (every 1000 years); then transforming the particles' coordinates into a reference frame rotating with the planet dominating the structure (Neptune); and finally treating each position as an individual particle, i.e. accumulating all the rotated particles' coordinates over the total lifetime of the sample particles. This leads to a three-dimensional time-weighted equilibrium density distributions that is "resampled" into a logarithmic one-dimensional radial grid which is the input for the radiative transfer code.

\subsection{Radial Density Distributions: Output from the Dynamical Models}

Figure 2 shows some of the resulting surface density distributions of dust. The main features are the following:

1. When no planets are present the dust density distribution is flat, as expected for a collisionless system with grains in circular orbits (Briggs 1962), and no large particles $(\beta<0.5)$ are found at distances larger than the apoastron of the parent bodies. But when planets are present, the surface density distribution deviates for a flat profile (see below) and gravitational scattering of dust by the giant planets is able to extend the disk beyond the boundaries set by radiation effects alone.

2. Depletion of dust inside the planet's orbit due to gravitational scattering by the planet. In the Solar System, depletion takes place in the inner 10 AU from gravitational scattering by Jupiter and Saturn. Inner cavities have been also inferred to exist in systems like $\beta$ Pic (20 AU), HR 4796A (30-50 AU), $\epsilon$ Eri (50 AU), Vega (80 AU) and Fomalhaut (125 AU) (Dent et al. 2000; Greaves, Mannings \& Holland 2000; Wilner et al. 2002 and Holland et al. 2003). Some of these systems are highly collisional, a regime where our modeling approach is not valid. The cavities, however, are possibly created by gravitational scattering with an inner planet.

3. Enhanced dust density in a ring outside the planet's orbit. This is produced by the trapping of particles in exterior MMRs with the planet. In the Solar System, the ring is between 35-50 AU and the resonant planet is Neptune. The trapping into MMRs can clearly be seen in the "equilibrium" semimajor axis distributions shown in Figure 3 (for the single-planet models) and Figure 10 in Moro-Martín \& Malhotra (2002) (for the Solar System models). 
4. The structure is more pronounced for larger particle sizes (smaller $\beta$ ) because the trapping in MMRs is more efficient when the drag forces are small. The boundary of the disk is less steep for smaller particles (larger $\beta$ ) compared to larger particles; this is because immediately after release from their parent bodies the orbits of the former are more strongly affected by radiation pressure, which tends to increase their eccentricity and semimajor axis.

The surface density distributions in Figure 2 show in some cases scattering at small astrocentric distances. In others cases, the particles drifting inward do not follow a flat surface density (instead it rises steeply). The presence of planets may explain part of these features, but from experience we know that some of this "noise" owes to our use of a logarithmic radial sampling to allow higher spatial resolution near the central star (where the high grain temperature implies a strong contribution to the SED). Where the radial shells are very small, a particle crosses many radial grids before its position is recorded; this, together with the fact that we are modeling the dynamical evolution of a small number of test particles (N 100), produces numerical "noise" due to small number statistics. For this reason, as described in $\S 2.2$, the radial density distributions that are used as input for the radiative transfer code will not take into account the numerical results at small astrocentric distances.

The depletion factor inside the planet's orbit is the percentage of particles that are ejected from the system, relative to the total number of particles. Because we are modeling sets of only 100 particles, and jovian-mass planets eject a significant fraction of these, the number of particles that drift inward is usually small and is subject to some uncertainty. We have several KB models with the same or similar initial conditions whose results indicate that a conservative estimate of the uncertainty in the depletion factor is $\sim 10 \%$ of the initial number of particles. Depletion factors are larger than $90 \%$ for the majority of the single-planet systems studied (and for most $\beta$ values), except for $1 \mathrm{M}_{\text {Jup }}$ at $1 \mathrm{AU}$ and $5 \mathrm{AU}$ for $\beta>0.025$, for which depletion factors are $\sim 60 \%-80 \%$ and $\sim 50 \%-80 \%$, respectively. This means that except for these two cases, the uncertainty due to the small number of particles studied makes the depletion factors obtained consistent with having an empty hole (i.e. $90 \% \pm 10 \%$ consistent with $100 \%$ depletion).

\subsection{Radial Density Distributions: Input for the Radiative Transfer Models}

The radial density distributions are uncertain interior to the planet's orbit to a $10 \%$ level. To account for this uncertainty, and to estimate the contribution of the particles trapped in the MMRs

to the SED of the disk, we will calculate and compare the SEDs that arise from three different types of surface density distributions (see Figure 4):

1. Empty Gap models: the surface density distribution accounts for the trapping of particles in the MMRs with the planet and the total depletion of particles interior to the planet's orbit, 
i.e. we assume that the "gap" 1 is empty (100\% depletion). The astrocentric distance of this "gap" is determined by the radius at which the surface density from the numerical results decreases by more than $90 \%$. Outside the gap, the surface density distribution follows the results from the dynamical models (large dots).

2. Partial Gap models: same as above but with the inner hole being $90 \%$ depleted in dust with respect to the disk without planets (instead of being totally empty, or $100 \%$ depletion). In this case, we are extrapolating the surface density of dust from a distance near the planet's orbit down to $0.01 \mathrm{AU}$, to account for the sublimation distance of the larger silicate grains. The detailed calculation of the sublimation distance is done by the radiative transfer code, and depends on the grain radius and chemical composition. The extrapolation is done assuming a flat surface density distribution, expected for a collisionless system with grains in circular orbits. The caveat is that when planets are present the dust grains that drift inward may have a non-zero eccentricity, so the surface density will not be exactly flat.

3. Analytical Gap models: the surface density distribution consists on a simple square profile, following the flat density distribution of the disk without planets, with an empty gap at the planet's position (i.e. it does not account for particles trapped in the MMRs).

The comparison of the SEDs that arise from the model with an empty gap and the model with a partial gap can teach us if the dynamical models are sufficient to distinguish the presence of planets of masses ranging from 1-10 $\mathrm{M}_{J u p}$ and semimajor axis of between 1-30 AU. If we find that the SEDs arising from these two models are significantly different, the number of particles studied (N 100) would not be sufficient, as it does not allow us to distinguish between an empty gap and a partial gap with $10 \%$ of particles left. The comparison of the SEDs that arise from the empty gap and the analytical gap models can teach us if the dynamical models are necessary, or whether it is adequate to assume a flat surface density distribution with a clean gap inward of the planet's location (ignoring the accumulation of dust particles in the MMRs).

The calculation of these radial density distributions is the most CPU demanding step, but it is independent of the rest of the steps outlined in the Introduction because the dynamical models and the resulting radial density profiles depend only on the parameter $\beta$. It is only a posteriori that we find the relationship between the particle size and composition and the $\beta$ value, with a single $\beta$ value corresponding to several combinations of grain size and composition (see Figure 5). Because of this degeneracy, our scheme allows enough flexibility to efficiently explore other grain chemistries without the need of recomputing the radial density profiles.

\footnotetext{
${ }^{1}$ In this paper a "gap" is an inner hole interior to the planet's orbit, not an angular depletion zone around the planet's orbit.
} 


\section{Correspondence between $\beta$ and Particle Size}

The quantity $\beta$ is the dimensionless ratio of the radiation pressure force and the gravitational force. For spherical grains and a solar type star, $\beta=5.7 \times 10^{-5} \mathrm{Q}_{p r} /(\rho a)$, where $\rho$ and $a$ are the density and radius of the grain in cgs units (Burns, Lamy \& Soter 1979), and $\mathrm{Q}_{p r}$ is the radiation pressure coefficient. Before using the radial density distributions as input for the radiative transfer code in WH03, we need to find the correspondence between the value of $\beta$ and the particle size, and this depends on the grain chemical composition. Detailed chemical analysis of young, massive circumstellar disks around T Tauri and Herbig Ae/Be stars show that the dust consists mainly of two distinct species: a silicate component and a carbonaceous component (Savage \& Mathis 1979; Draine \& Lee 1984 and Malfait et al. 2000). ISO spectroscopy of Herbig Ae/Be stars revealed several other chemical components, such as Fe (broad, weak emission feature at $24 \mu \mathrm{m}$; see Henning \& Stognienko 1996), FeO (broad emission features at $2125 \mu \mathrm{m}$; see Henning et al. 1995), and/or FeS (see Henning \& Stognienko 1996) and $\mathrm{H}_{2} \mathrm{O}$ ice (broad emission features between 40 and $80 \mu \mathrm{m}$; Warren 1984). The SEDs of debris disks measured so far, however, do not allow one to perform a comparably detailed chemical analysis. Mid-IR spectroscopy of $\beta$-Pic can be explained with dust grains composed of 55\% amorphous olivine, $35 \%$ amorphous pyroxen and 10\% crystalline olivine (see e.g. Pantin, Lagage \& Artymowicz 1997). The zodiacal light silicate feature can be matched by a mixture of amorphous forsterite/olivine, dirty crystalline olivine and a hydrous silicate (Reach et al. 2003). Based on these spectroscopic observations, the following chemical compositions were selected: $\mathrm{MgSiO}_{3}$ and $\mathrm{Mg}_{0.6} \mathrm{Fe}_{0.4} \mathrm{SiO}_{3}$ (Fe-poor and Fe-rich pyroxene), $\mathrm{MgFeSiO}_{4}$ and $\mathrm{Mg}_{1.9} \mathrm{Fe}_{0.1} \mathrm{SiO}_{4}$ (amorphous and crystalline olivine), and $\mathrm{C} 400$ and $\mathrm{C} 1000$ [400 K carbon modification (graphite-poor) and $1000 \mathrm{~K}$ carbon modification (graphite-rich), respectively]. This is a subset of the compositions studied in WH03; we refer to this paper for a justification of this selection and a description of the optical properties of these silicate and carbon species. The SEDs presented in this study correspond to disks composed of only one grain type (of the six listed above). A mixture of grains is more realistic, with the grain composition probably varying from source to source (see e.g. Reach et al. 2003). Because we are assuming that the disks are optically thin, so that the grains do not interact with each other, we can account for different mixtures by linearly combining the single grain composition SEDs into a final SED, using different contributing factors for each of the grain chemical compositions. This step will be taken in the future when using our models to simulate Spitzer observations.

For each selected chemistry, using Mie scattering theory and assuming that the grains are homogeneous spheres, we compute the grain optical parameters, needed to calculate dust absorption, reemission and scattering of radiation. We obtain $\mathrm{Q}_{p r}$ as a function of wavelength for a large number of particle sizes. The quantity $\mathrm{Q}_{p r}$ is a function of the grain complex refractive indexes $(n, k)$, the grain radius, and the wavelength of the incoming radiation. The refractive index for the silicates and carbonaceous materials are taken from Dorschner et al. (1995) and Jäger et al. 
(1998), respectively ${ }^{2}$. We then obtain the average of $\mathrm{Q}_{p r}$ integrated over the solar spectrum (Labs $\&$ Neckel 1968). This average is used to calculate the value of $\beta$, for each particular dust chemistry under consideration, and for a large number of particle sizes (see Figure 5). Finally, we select the particle size whose $\beta$ is closer to the $\beta$-value adopted in the dynamical models. One important feature to notice from Figure 5 is that, for a given particle size, the value of $\beta$ corresponding to carbonaceous and Fe-rich silicate grains is larger than that of Fe-poor silicates, because the former have a very high absorptive efficiency in the wavelength range on which the star emits. This is important because a small change in the abundance of carbonaceous and Fe-rich silicate material can make a very significant change in the level of the continuum emission.

The parameter study in WH03 showed that the shape of the SED is affected by the relative number of small grains, which is determined by the minimum $\left(a_{\min }\right)$ and maximum $\left(a_{\max }\right)$ grain size, and by the index $(q)$ of the power law size distribution $\left(n(a) d a=n_{0} a^{-q}\right)$ : an increase of net flux and the prominent emission features occur when $a_{\text {min }}$ is decreased and $q$ is increased. In this paper $a_{\min }=0.5-1.3 \mu \mathrm{m}$ (depending on composition), and is determined by the condition $\beta=0.5$, corresponding to particles that are forced into hyperbolic orbits as soon as they are released from their parent bodies. If the parent bodies' orbits have eccentricity $e$, ejection occurs for $\beta>0.5(1-e)$ and $\beta>0.5(1+e)$ for particle release at periastron and apoastron, respectively. We are therefore implicitly assuming that radiation pressure is the only process responsible for the minimum grain size, but in practice $a_{m i n}$ is also affected by collisional processes. The maximum grain size in our simulations is limited by the CPU time, as particles with very small $\beta$ s (0.00156 is our minimum value) have a very slow dynamical evolution. Depending on the chemical composition chosen, $a_{\max }=53-244 \mu \mathrm{m}$. Debris disks certainly contain larger "dust" particles, up to planetesimal size, but in the wavelength range considered grains larger than $\sim 1 \mathrm{~mm}$ will not contribute significantly to the SED, and the missing grains in the $53-240 \mu \mathrm{m}$ to $1 \mathrm{~mm}$ range only add an almost featureless continuum, as indicated in the study by WH03.

\section{Spectral Energy Distributions}

Once the particle size is known for each $\beta$ value and selected chemical composition, we use the surface density distributions as input for a radiative transfer code that calculates the emitted dust SED (plus the stellar scattered light). As in WH03, we assume that the disk is optically thin: only scattering, absorption and reemission of stellar radiation by dust grains are taken into account, neglecting multiple scattering and radiation and dust heating due to dust reemission. The dynamical models are only valid in a density regime that corresponds to optically thin disks. For the central star we use the solar SED published by Labs \& Neckel (1968), 0.2-100 $\mu \mathrm{m}$, extended by a blackbody SED $(\mathrm{T}=5800 \mathrm{~K})$ beyond $100 \mu \mathrm{m}$. The dust reemission and scattering are calculated at 500 logarithmically equidistantly distributed wavelengths between 5-340 $\mu \mathrm{m}$ (which includes

\footnotetext{
${ }^{2}$ The complex refractive indexes are available at http://www.astro.uni-jena.de/Laboratory/Database/odata.html.
} 
the wavelengths covered by Spitzer). We assume a disk mass of $10^{-10} \mathrm{M}_{\odot}$ and a distance of 50 pc. Note that our models (with and without planets) contain the same amount of disk mass. We are interested in studying how the structure created by the planets affects the shape of the SED, independent of the dust production rate. However, planetary perturbations can affect the dust production rate, possibly leading to more massive dust disks. This effect is not taken into account in our models, but will be considered in the future.

Figure 6 shows the SEDs that result from the Solar System models. Each color corresponds to the SED that arise from a single particle size disk (we only show three $\beta$ values of the 17 computed). Each panel corresponds to a particular grain chemical composition. In Figure 7 we compare the emission arising from the different compositions (keeping the particle size approximately constant). In agreement with WH03, the most important features shown in these figures are:

1. Emission is stronger for Fe-rich silicates $\left(\mathrm{Mg}_{0.6} \mathrm{Fe}_{0.4} \mathrm{SiO}_{3}\right.$ and $\left.\mathrm{MgFeSiO}_{4}\right)$ compared to Fepoor $\left(\mathrm{MgSiO}_{3}\right.$ and $\left.\mathrm{Mg}_{1.9} \mathrm{Fe}_{0.1} \mathrm{SiO}_{4}\right)$. This is due to the strong dependence of the UV-to-NIR absorption efficiency on the Fe content, which leads to higher grain temperatures at a given distance from the star as the Fe content increases.

2. Similarly to Fe-rich silicates, carbonaceous grains also lead to stronger but featureless emission (mainly adding a continuum).

3. The emission peak shifts to longer wavelengths as the particle size increases (or $\beta$ decreases). This is due to the fact that the turnover point beyond which the absorption efficiency decreases continuously increases with grain size.

4. The clearing of dust from the inner $10 \mathrm{AU}$ results in a loss of warm dust and is responsible for the decrease in the NIR/MIR region (compared to the case when no planets are present). The slight shift in the emission peaks indicate that some of this NIR/MIR emission is radiated at longer wavelengths (as the cleared particles are located further away from the star), but this is a very small effect because once the particles are set on hyperbolic orbits after their last gravitational encounter with the giant planet, they leave the system very quickly without contributing significantly to the emission. The net flux decreases because a larger fraction of the grains are further away from the star, so the fraction of stellar photons that the grains can absorb and later re-emit is diminished.

Similarly, but not shown here, we have calculated single particle size and single composition SEDs for the other planetary systems studied (i.e. the nine single-planet models and the system without planets). These SEDs show features similar to those described above for the Solar System.

As Figure 2 shows, the structure of the dust disk is significantly different depending on the particle size under consideration. The structure is more pronounced for larger particle sizes because the trapping in resonances is more efficient when the drag forces are small. However, it is expected that the dust production processes will favor the generation of small particles. The modeling of 
debris disk structure and SED should therefore take into consideration an appropriate range of particle sizes that can later be weighted and combined to emulate a particle size distribution. In Moro-Martín \& Malhotra (2003) we estimated the radial distribution of KB dust from our dynamical models and the KB dust production rate estimates from Landgraf et al. (2002). We showed that the dust particle size distribution in space is significantly changed from its distribution at production, due to the combined effects of radiation forces and the perturbations of the planets. Radiation forces alone change the differential size distribution from the (assumed) initial power law of index $q=3.5$ at production (corresponding to a fragmentation power law), to a shallower power law with $q \approx 2.5$, valid at distances smaller than the aphelion of the parent bodies. Planetary perturbations further affect the power law index because larger particles are more easily trapped in MMRs. With these results in mind, the single particle size and single composition SEDs for each planetary system (like those shown in Figure 6 for the Solar System) are weighted and combined in such a way that particle size distribution throughout the disk (not necessarily where the dust is generated) follows power laws of indexes $q=2.5,3.0$ and 3.5 (i.e each SED corresponds to a dust mass of $10^{-10} \mathrm{M}_{\odot}$ and is weighted in such a way that the total mass in each particle size bin follows a power law). Note that when collisional processes are considered in detail, the particle strengths are size-dependent, leading to a size-dependent $q$; and because the particle growth/collision processes depends on the radial distance from the star, $q$ will also be a function of radius. The trapping of particles in MMRs with the planets also adds a radial dependency to the power law index. Here, we will ignore these effects and consider a single power law to describe the particle size distribution at all distances from the star and for all particle sizes.

The top panel of Figure 8 shows that the flux is higher, specially in the NIR/MIR range, and the spectral features are more pronounced when the particle size distribution is steeper (i.e. there is a larger fraction of smaller grains). This is because small grains achieve higher temperatures. In WH03 it was found that because of this effect, the presence of a gap, and consequently the removal of warm grains, lead to a more pronounced decrease of flux in the NIR/MIR range when a larger fraction of smaller grains were present (steeper power law). Figure 8 (bottom) suggest exactly the opposite trend, as the ratio of the SEDs that arise from a system with a planet (using the empty gap models) to that from a system without a planet (i.e. without a "gap") is smaller for smaller power law indexes. The discrepancy between this result and the one in WH03 arises from the difference between the analytic density distributions used in WH03, and the density distributions used here; the latter showing a large difference between the system with planets and the system without planets, which is more pronounced for larger grains (smaller $\beta$ ) than for smaller grains (larger $\beta$; see Figure 2). This means that the difference between the mean disk temperatures in the system with planets and that of the system without planets is more pronounced when large particles are dominant, leading to more distinct differences in their corresponding SEDs. This illustrates the importance of combining numerical tools for the simulation of debris disk structure with a detailed radiative transfer code for the calculation of their emergent SEDs.

The SEDs that result after combining the different grain sizes are shown in Figure 9. Each panel 
corresponds to a different planetary system (indicated at the top). Figure 10 shows the ratios of the SEDs that arise from a system with a planet (using the empty gap models) to that from a system without a planet (i.e. without a "gap"). In the following, we refer to this ratio as $\mathrm{F}_{\text {planet }} / \mathrm{F}_{\text {no planet }}$, where $\mathrm{F}$ is the flux arising from the dust disk. Similarly, we show $\mathrm{F}_{\text {partial gap }} / \mathrm{F}_{\text {empty gap }}$ and $\mathrm{F}_{\text {empty gap }} / \mathrm{F}_{\text {analytical gap. }}$ The SED depends on the grain chemical composition. For example, Figure 9 shows that for carbonaceous and Fe-rich silicates grains, the minimum in the SED of a dust disk with Solar System-like planets is at $\lambda<8 \mu \mathrm{m}$, while for Fe-poor silicate grains the minimum shifts to longer wavelengths, $\lambda=10-25 \mu \mathrm{m}$. A similar effect is also found for the other single-planet systems studied. Similarly, the minimum of $\mathrm{F}_{\text {planet }} / \mathrm{F}_{\text {no planet }}$ occurs at $\lambda<10 \mu \mathrm{m}$ for carbonaceous and Fe-rich silicates grains, and $\sim 15 \mu \mathrm{m}$ for Fe-poor silicate grains, i.e., the wavelength range where the difference between the SED arising from a disk with Solar System-like planets and that of a disk without planets is the largest depends on the chemical composition of the grains. As it can be seen from Figure 10, in many cases the largest difference between $\mathrm{F}_{\text {planet }}$ and $\mathrm{F}_{\text {no planet }}$ occurs at wavelengths where the photospheric emission from the star dominates, making the photospheric subtraction critical in the analysis of observed SEDs in terms of planetary architectures.

Figure 10 shows that for a planet at 1 AU, the differences between the "empty gap" and the "analytical gap" models are large, with $\mathrm{F}_{\text {empty gap }} / \mathrm{F}_{\text {analytical gap }}$ up to 100 for $1 \mathrm{M}_{\text {Jup }}$, and 30 for $10 \mathrm{M}_{\text {Jup }}$ (dashed lines). This is due the fact that the "analytical gap" models follow a square profile, while the dynamical models contain a large number of particles accumulated in the MMRs with the planet (see Figure 2 and note that scale is logarithmic). These particles, being at small astrocentric distances, are hot and contribute very significantly to the SED. We can conclude that dynamical simulations are necessary to model the SEDs of debris disks in the presence of planets at small semimajor axis (hot Jupiters), because the enhancement of particles at the MMRs dominates the emission. A consequence of this is that it should be possible to distinguish observationally between a simple square profile for the surface density of the dust disk, as that created by a stellar wind or by the interaction of the dust grains with ambient gas, from the surface density created by the dynamical interactions with a massive planet.

Figure 10 also shows that for a planet at $1 \mathrm{AU}$, the ratio $\mathrm{F}_{\text {planet }} / \mathrm{F}_{\text {no planet }}$ (solid lines) is greater than 1 for $\lambda=8-60 \mu \mathrm{m}$ for carbonaceous and Fe-rich silicate grains, and $\lambda=20-80 \mu \mathrm{m}$ for Fe-poor silicate grains. Even though the disk with a planet has an inner hole, it can be up to 3 times brighter than the disk without a planet. This is because the particles accumulated in the MMRs contribute importantly to the SED. The system with $10 \mathrm{M}_{J u p}$ at $1 \mathrm{AU}$, however, is not significantly brighter than the system without planet. This does not mean that the analytical square profiles are sufficient for $10 \mathrm{M}_{J u p}$ at $1 \mathrm{AU}$, because as we saw above, $\mathrm{F}_{\text {empty gap }} / \mathrm{F}_{\text {analytical gap }} \sim 30$. The ratio $\mathrm{F}_{\text {planet }} / \mathrm{F}_{\text {no planet }}$ is very close to 1 , for $\lambda>80 \mu \mathrm{m}$ for $1 \mathrm{M}_{\text {Jup }}$ at $1 \mathrm{AU}$, and $\lambda>24 \mu \mathrm{m}$ for $10 \mathrm{M}_{\text {Jup }}$ at $1 \mathrm{AU}$. In this wavelengths ranges, either the effect of the particles accumulated in the resonances is not important, or their effect on the SED is balanced by the depletion of hot grains close to the star.

The "partial gap" and "empty gap" models are very similar for $1 \mathrm{M}_{\text {Jup }}$ at $1 \mathrm{AU}\left(\mathrm{F}_{\text {partial gap }} / \mathrm{F}_{\text {empty gap }}\right.$ 
$\sim 1$; dotted lines in Figure 10). For a $10 \mathrm{M}_{\text {Jup }}$ planet at $1 \mathrm{AU}, \mathrm{F}_{\text {partial gap }} / \mathrm{F}_{\text {empty gap }} \sim 3$, for $\lambda<20 \mu \mathrm{m}$. But in this case, our models indicate that the number of particles that drift inward is probably 0 , with an uncertainty smaller than $10 \%$, which means that $\mathrm{F}_{\text {partial gap }} / \mathrm{F}_{\text {empty gap }}$ is probably overestimating the uncertainties in the prediction of the SED. We conclude from the small $\mathrm{F}_{\text {partial gap }} / \mathrm{F}_{\text {empty gap }}$ values that the uncertainties in our dynamical simulations, due to the small number of particles studied, do not affect the modeling of the SEDs arising from dust disks with a planet at $1 \mathrm{AU}$. In other words, the number of particles in our simulations ( $\mathrm{N}$ 100) is sufficient to model these systems with close-in planets.

As we saw before, for $1 \mathrm{M}_{J u p}$ at $1 \mathrm{AU}$ the accumulation of dust grains in the MMRs with the planet can increase the flux up to a factor of 100 compared to the flux arising from a disk with a simple square profile. For $1 \mathrm{M}_{J u p}$ at $5 \mathrm{AU}$, the maximum $\mathrm{F}_{\text {empty gap }} / \mathrm{F}_{\text {analytical gap }}$ decreases from $\sim 100$ to $\sim 30$, and therefore the accumulation into the MMRs is not as important as at $1 \mathrm{AU}$ (the particles are colder and their contribution is less dominant). But the difference between the "partial gap" and "empty gap" models is more pronounced at $5 \mathrm{AU}\left(\mathrm{F}_{\text {partial gap }} / \mathrm{F}_{\text {empty gap }} \sim 3-10\right.$ for $1 \mathrm{M}_{\text {Jup }}$ and 10-30 for $10 \mathrm{M}_{\text {Jup }}$ ) than at $1 \mathrm{AU}\left(\mathrm{F}_{\text {partial gap }} / \mathrm{F}_{\text {empty gap }} \sim 1\right.$ for $1 \mathrm{M}_{\text {Jup }}$ and 3 for $\left.10 \mathrm{M}_{\text {Jup }}\right)$. [The number of particles that drift inward for $10 \mathrm{M}_{J u p}$ at $5 \mathrm{AU}$ is probably 0 and not subject to the $10 \%$ uncertainty, so the factor of 10-30 is probably overestimated.] We conclude that the dynamical models are necessary to study the SEDs arising from systems with planets of $1-10 \mathrm{M}_{\text {Jup }}$ at $5 \mathrm{AU}$, because by not considering the particles accumulated in the MMRs, the SED can be underestimated by a factor of 30 . But unlike the $1 \mathrm{AU}$ models, the number of particles that we have used in our simulations $(\mathrm{N} \sim 100)$ is not sufficient because a $10 \%$ uncertainty in depletion factor yields to a factor of 3-10 and 10-30 (overestimated) in flux for 1 and $10 \mathrm{M}_{J u p}$, respectively.

The models at $30 \mathrm{AU}$ represent a system with a narrow ring of dust producing planetesimals just outside the planet's orbit. If a fraction of the dust particles drift inward, even if it is small, the shape of the SED is similar to the one arising from a system without planets. This is because like in the models without planets, there is no structure for a wide range of astrocentric distances. If the particles are held back (by trapping in resonances and gravitational scattering), the SED shows a large deficit in the mid-IR flux (whose wavelength depend on the location of the ring) that makes it very distinct from the SED from a system without planets (see large minimum for $\mathrm{F}_{\text {planet }} / \mathrm{F}_{\text {no planet }}$ in Figure 10$)$.

The "empty gap" and "analytical gap" models are very similar when the planet is at 30 AU, i.e. the accumulation of particles in the MMRs do not dominate the shape of the SED for planets at this distance. However, the "partial gap" models are very different from the "empty gap" models, with the $10 \%$ uncertainty in the number of particles that drift inward leading to a factor of 100-3000 difference in flux $\left(\mathrm{F}_{\text {partial gap }} / \mathrm{F}_{\text {empty gap }} \sim 100\right.$, for $1 \mathrm{M}_{\text {Jup }}$ and $\mathrm{F}_{\text {partial gap }} / \mathrm{F}_{\text {empty gap }} \sim 1000-3000$, for $10 \mathrm{M}_{\text {Jup }}$ ). Because the SED is very sensitive to the number of particles that drift inward, this number needs to be determined precisely by dynamical models; the large factors indicate that the number of particles in our simulations $(\mathrm{N} \sim 100)$ is not sufficient to study systems with planets of $1-10 \mathrm{M}_{\text {Jup }}$ at $30 \mathrm{AU}$. 
The ratio $\mathrm{F}_{\text {planet }} / \mathrm{F}_{\text {no planet }}$ can reach 0.3 for $1 \mathrm{M}_{J u p}$ at $1 \mathrm{AU}$, and 0.1 for $10 \mathrm{M}_{J u p}$ at $1 \mathrm{AU}$. This decrease is due to the fact that for the more massive planet the gap is larger $\left(\mathrm{r}_{\text {gap }} \sim 0.8 \mathrm{AU}\right.$ for $1 \mathrm{M}_{\text {Jup }}$, and $\sim 1.6 \mathrm{AU}$ for $10 \mathrm{M}_{\text {Jup }}$; see Figure 4 ), and more empty (larger number of particles are ejected). The different depletion factors explain why a disk with $1 \mathrm{M}_{J u p}$ at $1 \mathrm{AU}$ (5 AU) is brighter than a disk with $10 \mathrm{M}_{J u p}$ at $1 \mathrm{AU}(5 \mathrm{AU}$ ) by a factor of $3-10$ (for $\lambda<10 \mu \mathrm{m}$ for carbonaceous and Fe-rich silicate grains; and $\lambda$ between 10-24 $\mu \mathrm{m}$ for Fe-poor silicate grains). The different gap radius makes the disk with a $1 \mathrm{M}_{\text {Jup }}$ at $1 \mathrm{AU}$ be 100 (3000) times brighter than a disk with $1 \mathrm{M}_{\text {Jup }}$ at $5 \mathrm{AU}(30 \mathrm{AU}$ ) (for $\lambda<24 \mu \mathrm{m}$, if the grains have carbonaceous and Fe-rich silicate composition; and $\lambda<50 \mu \mathrm{m}$, if they have Fe-poor silicate composition).

\section{Predicted Spitzer Broadband Colors}

We have calculated expected Spitzer broadband colors. In principle, the SEDs in Figure 9 should contain all the information given by the color-color diagrams. However, the advantage of these diagrams is that one can compare easily the results arising from many different models, allowing to explore more efficiently the parameter degeneracies in the model SEDs. In particular, we are interested in exploring the effects of planet mass and location, particle size distribution and composition, and the depletion factor inside the gap cleared by the planet.

The central bandpass wavelengths are $4.51 \mu \mathrm{m}$ and $7.98 \mu \mathrm{m}$ (IRAC), and $23.68 \mu \mathrm{m}, 71.42 \mu \mathrm{m}$ and $155.9 \mu \mathrm{m}$ (MIPS) ${ }^{3}$. In addition, we have integrated the SEDs using square profiles centered at $13.2 \mu \mathrm{m}$ and $32.5 \mu \mathrm{m}$, with widths of $1.6 \mu \mathrm{m}$ and $5.0 \mu \mathrm{m}$ (for the IRS observations). These widths are chosen to avoid the bad segments of the IRS instrument and the long-wavelength tail of the $10 \mu \mathrm{m} \mathrm{SiO}$ feature. Figure 11 shows five different combinations of color-color diagrams (one per row). Each panel corresponds to a different grain chemical composition (only three are shown). The different symbols correspond to different planetary systems: the symbol shape indicates the planet semimajor axis and the the symbol color indicates the planet mass, the power law index for the particle size distribution, and whether the gap is empty or partially filled.

The types of planetary systems that can be distinguishable from one another based on their Spitzer colors are listed in Table 1. The main results are the following:

1. The colors considered here can be used to diagnose the location of the planet (in the 1-30 AU range) and the absence/presence of planets, if the gap cleared by the planet is depleted by more than $90 \%$.

2. Except for one particular case, in general it is not possible to diagnose the mass of the planet (in the $1-10 \mathrm{M}_{\text {Jup }}$ range) based on these colors.

\footnotetext{
${ }^{3}$ Spectral responses are available at http://ssc.spitzer.caltech.edu/irac/spectral_response.html and http://ssc.spitzer.caltech.edu/mips/spectral_response.html.
} 
3. If the disks are composed of carbonaceous grains (C400 and C1000), the different planetary systems considered have indistinguishable Spitzer colors, i.e. the higher the carbonaceous content the more difficult it is to diagnose planetary systems from their colors.

4. If instead of "empty gap" models we consider "partial gap" models, where $10 \%$ of the particles drift inward, most of the planetary systems in Table 1 become indistinguishable from one another. It is important to keep this in mind when looking at the results in Table 1 involving planets at 5 or $30 \mathrm{AU}$, as in these cases our dynamical models do not contain enough particles to determine precisely how many of them drift inward (so we are subject to a 10\% uncertainty).

5. In some cases (see Table 1), two planetary systems that are distinguishable for one index of the power law for the grain size distribution, are not distinguishable when using a different index $(q=2.5$ versus $q=3.5)$. This, together with the fact that different compositions yield different results (see e.g. how different are the colors for $\mathrm{MgSiO}_{3}$ grains compared to the other compositions), complicates the analysis of the colors, as the particle size distribution and chemical composition are not known.

6. $24 \mu \mathrm{m} / 32 \mu \mathrm{m}$ and $70 \mu \mathrm{m} / 160 \mu \mathrm{m}$ colors are not useful to distinguish among the planetary systems considered in this study $\left(1-10 \mathrm{M}_{\text {Jup }}\right.$ at $\left.1-30 \mathrm{AU}\right)$.

\section{SED Probability Distribution}

The previous analysis describes how the SED is affected by variations of specific parameters (e.g. the presence/absence of planets, the chemical composition of the dust, and the particle size distribution). Because in many cases these parameters are unknown, in this section we consider the degeneracy that is created by the entire suite of parameters. In order to do that, we have selected the four radial density distributions shown in the upper left panel of Figure 2. They correspond to a disk with a $1 \mathrm{M}_{\text {Jup }}$ planet located at $1 \mathrm{AU}$, and four different values of $\beta$ (or grain sizes): $0.00156,0.0125,0.1$ and 0.4 . For each of one of these four distributions, we considered 21 different depletion factors: from $80 \%$ to $100 \%$ (empty gap) in increments of $1 \%$. We then "shifted" each one of these $4 \times 21$ distributions in the radial coordinate, to simulate the radial density distributions that would arise if the planet is located at a larger semimajor axis. We considered 36 planet locations between $1 \mathrm{AU}$ and $30 \mathrm{AU}$. This adds up to a total of $4 \times 21 \times 36$ different radial density distributions. Although these distributions are not exactly the ones that would result from the dynamical models (if we were to run $4 \times 21 \times 36$ different disk models), a comparison with the results at $5.2 \mathrm{AU}$ and $30 \mathrm{AU}$ shows that they are a reasonable approximation, as they include the main features: a range of dust depletion factors inside the orbit of the planet, and accumulation of particles in the MMRs with the planet. For each one of these distributions, and selecting one grain mineralogy, we used the radiative transfer code to calculate its SED. The SEDs from the four different grain sizes (or $\beta$-values) are combined using 11 different power laws indexes (from 2.5 to 3.5, in increments of 0.1), 
resulting in a total of $21 \times 36 \times 11$ SEDs. We repeated this procedure for the six different chemical compositions considered in our study. The results are shown in Figures 12 and 13.

If the dominant grain composition is known, Figure 12 indicates that, for the six compositions considered, the shape of the SED of a disk with embedded planets is very distinct from the SED of a disk without planets. The main difference, as mentioned before, is the decrease of the near-midIR flux $(<40 \mu \mathrm{m})$, due to the clearing of particles inside the planet's orbit. Observations at long wavelengths $(>40 \mu \mathrm{m})$ are important to determine the shape of the SED, so we can distinguish whether the decreased near-mid-IR flux is due to a region depleted of dust, or to an overall less massive disk. However, if the dominant grain composition is unknown, Figure 13 indicates that it is not possible to distinguish between a disk with planets and a disk without planets. This illustrates the importance of obtaining spectroscopy observations able to constrain the grain chemical composition of the dust. Regardless of the chemical composition, there are two regions where the dispersion of the SEDs is small ( $\sim 1$ order of magnitude in flux). As expected, one is at the longest wavelengths $(>250 \mu \mathrm{m})$, where the emission is dominated by the largest grains, and therefore the spectral features are not as important (except for crystalline olivine); and the other region is at $\sim 60 \mu \mathrm{m}$ (as was also pointed out by WH03). If the disk and dust grain properties are unknown, these two regions are the most diagnostic for the determination of the total disk mass.

For each one of these SEDs we have calculated expected Spitzer broadband colors. The results are shown in Figure 14. For carbonaceous grains, the colors from a disk with embedded planets are indistinguishable from those of a disk without planets (as was pointed out in $\S 5$ ). The same happens for Fe-rich silicate grains, except for $70 \mu \mathrm{m} / 160 \mu \mathrm{m}$, where we could marginally distinguish between a disk with planets and a disk without planets (but only for certain planetary configurations). However, if Fe-poor silicate grains dominate, Figure 14 indicates that the colors can indeed be used to diagnose the presence or absence of embedded planets, in particular, $8 \mu \mathrm{m} / 13.2 \mu \mathrm{m}, 24 \mu \mathrm{m} / 70 \mu \mathrm{m}$ and $24 \mu \mathrm{m} / 32 \mu \mathrm{m}$.

\section{Conclusions and Future Work}

In anticipation of future observations of spatially unresolved debris disks with Spitzer, we are interested in studying how the structure carved by planets affects the shape of the disk's SED, and consequently if the SED can be used to infer the presence of planets. We numerically calculate the equilibrium spatial density distributions of dust disks composed of different grain sizes, originated by a belt of planetesimals similar to the $\mathrm{KB}$ and in the presence of interior giant planets in different planetary configurations. A radiative transfer code is used to generate their corresponding SED for a representative sample of grain chemical compositions. The goal is to find the main parameter degeneracies in the model SEDs and the distinguishing characteristics between the SEDs of different planetary configurations.

In practice, the modeling of an observed SED is done by using simple analytical surface density 
distributions defined by parameters that can be varied to fit the observations. However, these parameters cannot be chosen arbitrarily, independently from the SED of the embedded star or the dust grain properties. A self-consistent combination of numerical models for the simulation of debris disk dust density distributions (that take into account the interplay between the central star SED, the grain properties and the dust dynamics), and a radiative transfer code is needed for the calculation of the dust disk SED. Our models indicate that for close-in planets (1AU), an important parameter to consider is the enhancement factor in a ring-like structure located outside the planet's orbit, and related to the number of particles accumulated in the MMRs. Hot Jupiters can trap dust particles in MMRs at small astrocentric distances. These particles are hot and can have an important contribution to the SED. Trapping in resonances can therefore make the disk to look brighter, facilitating its detection, but it also makes a disk with an inner planet less distinguishable from a disk without planet, as the clearing of hot dust inside the planet's orbit (and its corresponding decrease of the near-mid-IR flux) is compensated by the trapping of particles in MMRs. For planets at larger semimajor axis (5 AU and $30 \mathrm{AU}$ ), the important parameter to consider is the density drop interior to the planet's orbit, related to the fraction of particles that are able to drift inward. These two parameters, describing the density enhancement and the density drop, depend on the mass and location of the planet, and can only be estimated using dynamical simulations. We conclude that: (1) dynamical models are necessary to study the SEDs arising from debris disk systems with embedded planets of $1-10 \mathrm{M}_{\text {Jup }}$ at 1-30AU; and (2) the number of particles in the dynamical simulations presented here $(\mathrm{N} \sim 100)$ is sufficient to study systems with planets at $1 \mathrm{AU}$, but for the study of planets at $5 \mathrm{AU}$ and $30 \mathrm{AU}$ we need to increase the number of particles in our simulations in order to improve the statistical uncertainty in the number of particles that drift inward to better than $10 \%$.

The SED of the dust disk depends on the grain properties (chemical composition, density and size distribution) and the mass and location of the perturbing planet. The SED of a debris disk with interior giant planets is fundamentally different from that of a disk without planets, the former showing a significant decrease of the near/mid-IR flux due to the clearing of dust inside the planet's orbit. The SED is particularly sensitive to the location of the planet, i.e. to the area interior to the planet's orbit that is depleted in dust (see Figure 15). However, there are some degeneracies that can complicate the interpretation of the SED in terms of planet location. For example, the SED of a dust disk dominated by Fe-poor silicate grains has its minimum at wavelengths longer than those of a disk dominated by carbonaceous and Fe-rich silicate grains. Because the SED minimum also shifts to longer wavelengths when the gap radius increases (owing to a decrease in the mean temperature of the disk), we note that there might be a degeneracy between the dust grain chemical composition and the semimajor axis of the planet clearing the gap. For an example, notice the similarities in the shape of the SEDs arising from a dust disk with a $3 \mathrm{M}_{J u p}$ planet at 1 $\mathrm{AU}$ and dominated by $\mathrm{MgSiO}_{3}$ grains, and a disk with a $3 \mathrm{M}_{J u p}$ planet at $30 \mathrm{AU}$ and dominated by $\mathrm{MgFeSiO}_{4}$ grains (see Figure 16). This illustrates the importance of obtaining spectroscopy observations able to constrain the grain chemical composition, and/or high resolution images, able to spatially resolve the disk. 
We saw that for planets at 5 and $30 \mathrm{AU}$, the difference in the SED arising from a disk with an empty gap interior to the planet's orbit, and a disk with an inner hole that is $90 \%$ depleted, is large. This means that the SED is very sensitive to the depletion factor inside the gap. Because this depletion factor depends largely on the planet mass, in principle one should be able to use SEDs to diagnose masses of planets at large astrocentric distances. To do that we would need to increase the number of particles in our dynamical simulations. Based on the depletion factors obtained from our models, we expect that the planetary masses that one would be able to study with this method would range from $>1 \mathrm{M}_{N e p}$ to $3 \mathrm{M}_{J u p}$. Anything larger than $3 \mathrm{M}_{J u p}$ would create an almost empty gap, being indistinguishable from one another. A $1 \mathrm{M}_{N e p}$ planet at $30 \mathrm{AU}$ ejects $<10 \%$ of the particles, so the efect of a less massive planet would probably be difficult to detect. At $1 \mathrm{AU}$, the SED of the disk seems to be more insensitive to the depletion factor (because as mentioned above, its effect on the SED is balanced by the particles in the MMRs). This means that it would be difficult to diagnose the mass of a close-in planet based on the SED of the dust disk.

The gaps and azimuthal asymmetries observed in high resolution images of debris disks suggest that giant planets may be present in these systems. Because debris disk structure is sensitive to a wide range of planet semimajor axis, complementing a parameter space not covered by radial velocity and transit surveys (sensitive only to close-in planets), the study of the disk structure can help us learn about the diversity of planetary systems. Even when spatially resolved images of the disk are not available, we have seen that its SED may contain the signatures of the underlying planets. The SED can therefore be a valuable tool for detecting and even constraining the mass and location of the planet.

Once interesting Spitzer targets have been identified, the next step will be to obtain highsensitivity and high-spatial resolution images in scattered light and/or thermal emission (using e.g. $L B T$, JWST, Sofia, ALMA or Safire). Of particular interest are the longer wavelengths, where observations can constrain the amount of material further away from the planet, and where the emission of the larger dust particles, the ones that show more prominent structure, dominate. If one could obtain observations that spatially resolve the disk, the dynamical models could allow us to locate the perturbing planet. Then we could compare the information derived from the SED alone to that derived from the resolved image. This is important for the understanding of the limitations of the characterization of planetary architectures based on spatially unresolved debris disks only. Also, by obtaining resolved images in one or more wavelength we can break the degeneracy expected from the analysis of the disk SED. In anticipation of these spatially resolved observations, we have started working on the modelling of the brightness density distributions arising from debris disks in the presence of different planetary configurations.

\section{Acknowledgments}

We thank Hal Levison for providing the SKEEL computer code, and Dana Backman, Martin Cohen, Lynne Hillenbrand, Jonathan Lunine, Michael Meyer, Alberto Noriega-Crespo and Steve 
Strom for useful discussion. We also want to thank the referee for her/his careful reading of the manuscript. This work is part of the Spitzer FEPS Legacy project (http://feps.as.arizona.edu). We acknowledge NASA for research support (contracts 1224768 and 1407 administered by JPL and

grants NNG04GG63G, NAG5-11661 and NAG5-11645) and IPAC and the Spitzer Science Center for providing access to their facilities. S. Wolf was also supported through the DFG Emmy Noether grant WO 857/2-1.

\section{REFERENCES}

Backman, D.E. \& Paresce, F. 1993, Protostars and Planets III (ed. E.H. Levy \& J.I Lunine, Tucson: Univ. of Arizona Press), 1253

Beichman C.A., Bryden, G., Rieke, G.H. et al., in preparation, 2005

Briggs, R.E. 1962, AJ, 67, 710

Burns, J.A., Lamy, P.L., Soter, S. 1979, Icarus, 40, 1

Dent, W.R.F., Walker, H.J., Holland, W.S. \& Greaves, J.S. 2000, MNRAS, 314, 702

Dorschner, J., Begemann, B., Henning, Th., Jäger, C. \& Mutschke, H. 1995, A\&A, 300, 503

Draine, B.T. \& Lee, H.M. 1984, ApJ, 285, 89

Gorlova, N., Padgett, D.L., Rieke, G.H. et al. 2004, ApJS, 154, 448

Greaves, J.S., Holland, W.S., Moriarty-Schieven, G. et al. 1998, ApJ, 506, 133

Greaves, J.S., Mannings, V. \& Holland, W.S. 2000, Icarus, 143, 155

Greaves, J.S. \& Wyatt, M.C. 2003, MNRAS, 345, 1212

Greaves, J.S., Holland, W.S., Jayawardhana, R., Wyatt, M.C. \& Dent,W.R.F 2004, MNRAS, 348, 1097

Habing, H.J., Dominik, C., Jourdain de Muizon, M. et al. 2001, A\&A, 365, 545

Henning, Th., Begemann, B., Mutschke, H., \& Dorschner, J. 1995, A\&AS, 112, 143

Henning, Th., \& Stognienko, R. 1996, A\&A, 311, 291

Holland, W.S. et al. 2003, ApJ, 582, 1141

Jäger, C., Mutschke, H. \& Henning, Th. 1998, A\&A, 332, 291

Kuchner, M.J., Holman, M.J. 2003, ApJ, 588, 110 
Labs, D. \& Neckel, H. 1968, ZA, 69, 1

Landgraf, M., Liou, J-C., Zook \& H.A, Grün, E. 2002, AJ, 123, 2857

Liou, J.C., Zook, H.A. \& Dermott, S.F. 1996, Icarus, 124, 429

Liou, J.C. \& Zook, H.A., 1999, AJ, 118, 580

Malfait, K., Waelkens, C., Bouwman, J., De Koter, A. \& Waters, L.B.F.M. 1999, A\&A, 345, 181

Malhotra, R., Duncan, M.J., Levison, H.F. 2000, Protostars and Planets IV (eds Mannings, V., Boss, A.P., Russell, S. S.), 1231

Moro-Martín, A. \& Malhotra, R. 2002, AJ, 124, 2305

Moro-Martín, A. \& Malhotra, R. 2003, AJ, 125, 2255

Mouillet, D., Larwood, J.D., Papaloizou, J.C.B. \& Lagrange, A.M. 1997, MNRAS, 292, 896

Ozernoy, L.M., Gorkavyi, N.N., Mather, J.C. \& Taidakova, T.A. 2000, ApJ, 537, 147

Pantin, E., Lagage, P.O. \& Artymowicz, P. 1997, A\&A, 327, 1123

Quillen, A.C. \& Thorndike, S. 2002, ApJL, 2002, 578, 149

Reach, W.T., Morris, P., Boulanger, F. \& Okumura, K. 2003, Iracus, 164, 384

Rieke et al. 2004, in press

Roques, F., Scholl, H., Sicardy, B. \& Smith, B.A. 1994, Icarus, 108, 37

Savage, B.D. \& Mathis, J.S. 1979, ARA\&A, 17, 73

Spangler, C., Sargent, A.I. \& Silverstone, M.D. 2001, ApJ, 555, 932

Weinberger, A.J., Becklin, E.E. \& Zuckerman, B. 2003, ApJ, 584, L33

Wilner, D.J, Holman, M.J., Kuchner, M.J. \& Ho, P.T.P 2002, ApJ, 569, L115

Wolf, S. \& Hillenbrand, L.A. 2003, ApJ, 596, 603

Warren, S. G. 1984, Appl. Opt., 23, 1206

Wyatt, M.C., Dermott, S.F. \& Telesco, C.M., 1999, ApJ, 527, 918

Wyatt, M.C. \& Dent, W.R.F. 2002, MNRAS, 334, 589 
Table 1. Planetary Systems with Distinct Colors

\begin{tabular}{|c|c|c|c|}
\hline Composition & $4 \mu \mathrm{m} / 8 \mu \mathrm{m}$ & $8 \mu \mathrm{m} / 13 \mu \mathrm{m}$ & $8 \mu \mathrm{m} / 24 \mu \mathrm{m}$ \\
\hline $\mathrm{MgSiO}_{3}$ & $1,5,30 \mathrm{AU}-\mathrm{no} \mathrm{pl}$ & $\begin{array}{c}\text { 1Jup1AU-1Jup30AU } \\
\text { 1Jup1AU-1Jup5AU } \\
\text { 1AU-30AU } \\
5 \mathrm{AU}-\mathrm{nopl}\end{array}$ & $\begin{array}{c}30 \mathrm{AU}-\mathrm{no} \mathrm{pl} \\
1,5 \mathrm{AU}-30 \mathrm{AU}\end{array}$ \\
\hline $\mathrm{Mg}_{0.6} \mathrm{Fe}_{0.4} \mathrm{SiO}_{3}$ & $\begin{array}{c}1 \mathrm{AU}-5 \mathrm{AU}^{\mathrm{a}} \\
1 \mathrm{AU}-30 \mathrm{AU} \\
5 \mathrm{AU}-\mathrm{no} \mathrm{pl}{ }^{\mathrm{a}} \\
30 \mathrm{AU}-\mathrm{no} \mathrm{pl} \\
1 \mathrm{Jup} 5-10 \mathrm{Jup} 5^{\mathrm{b}}\end{array}$ & 1,5AU,no pl-30AU & \\
\hline $\mathrm{MgFeSiO}_{4}$ & $1 \mathrm{AU}-30 \mathrm{AU} \mathrm{b}^{\mathrm{b}}$ & & \\
\hline $\mathrm{Mg}_{1.9} \mathrm{Fe}_{0.1} \mathrm{SiO}_{4}^{\mathrm{a}}$ & & 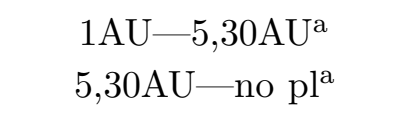 & \\
\hline
\end{tabular}

List of planetary systems with distinct Spitzer colors. Notation: "1,5AU$30 \mathrm{AU}$ " means that the models with the planet at 1 or $5 \mathrm{AU}$ are distinguishable from the models with the planet at $30 \mathrm{AU}$. $1 \mathrm{Jup} 1$ is a $1 \mathrm{M}_{J u p}$ planet at $1 \mathrm{AU}$. All results refer to "empty gap" models.

a only valid for $q=2.5$

${ }^{\mathrm{b}}$ only valid for $q=3.5$ 
Table 2. Planetary Systems with Distinct Colors

\begin{tabular}{lcc}
\hline \hline Composition & $13 \mu \mathrm{m} / 24 \mu \mathrm{m}$ & $24 \mu \mathrm{m} / 70 \mu \mathrm{m}$ \\
\hline $\mathrm{MgSiO}_{3}$ & $\begin{array}{l}1,5 \mathrm{AU}-30 \mathrm{AU} \\
30 \mathrm{AU}-\mathrm{no} \mathrm{pl}\end{array}$ & Jup1-1Jup30 \\
& & 1Jup1-1Jup30 \\
$\mathrm{MgFeSiO}_{4}$ & & \\
\hline
\end{tabular}




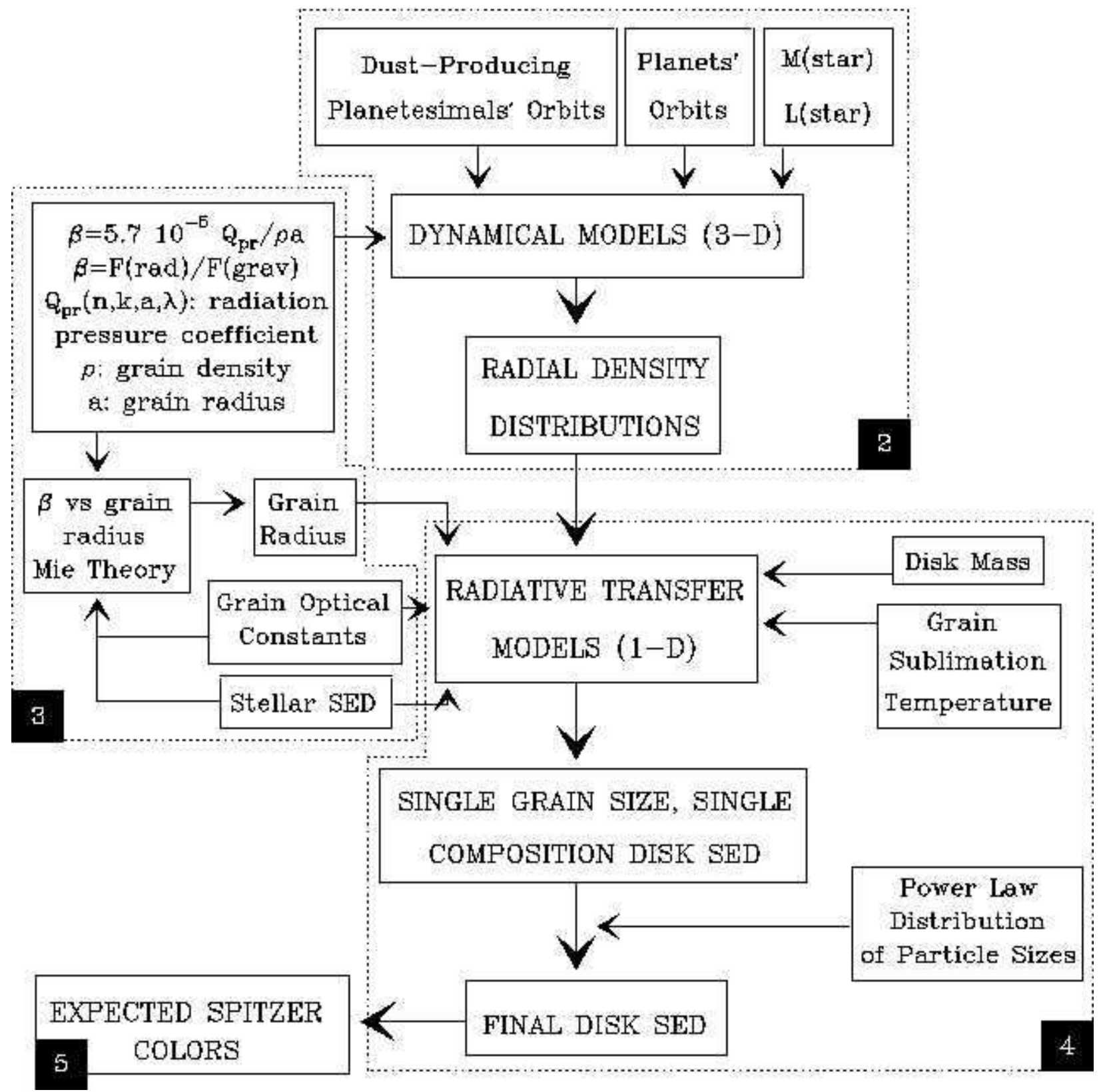

Fig. 1. - Schematic diagram showing the steps of the modeling approach. The numbers inside the black squares indicate the relevant sections in this paper 

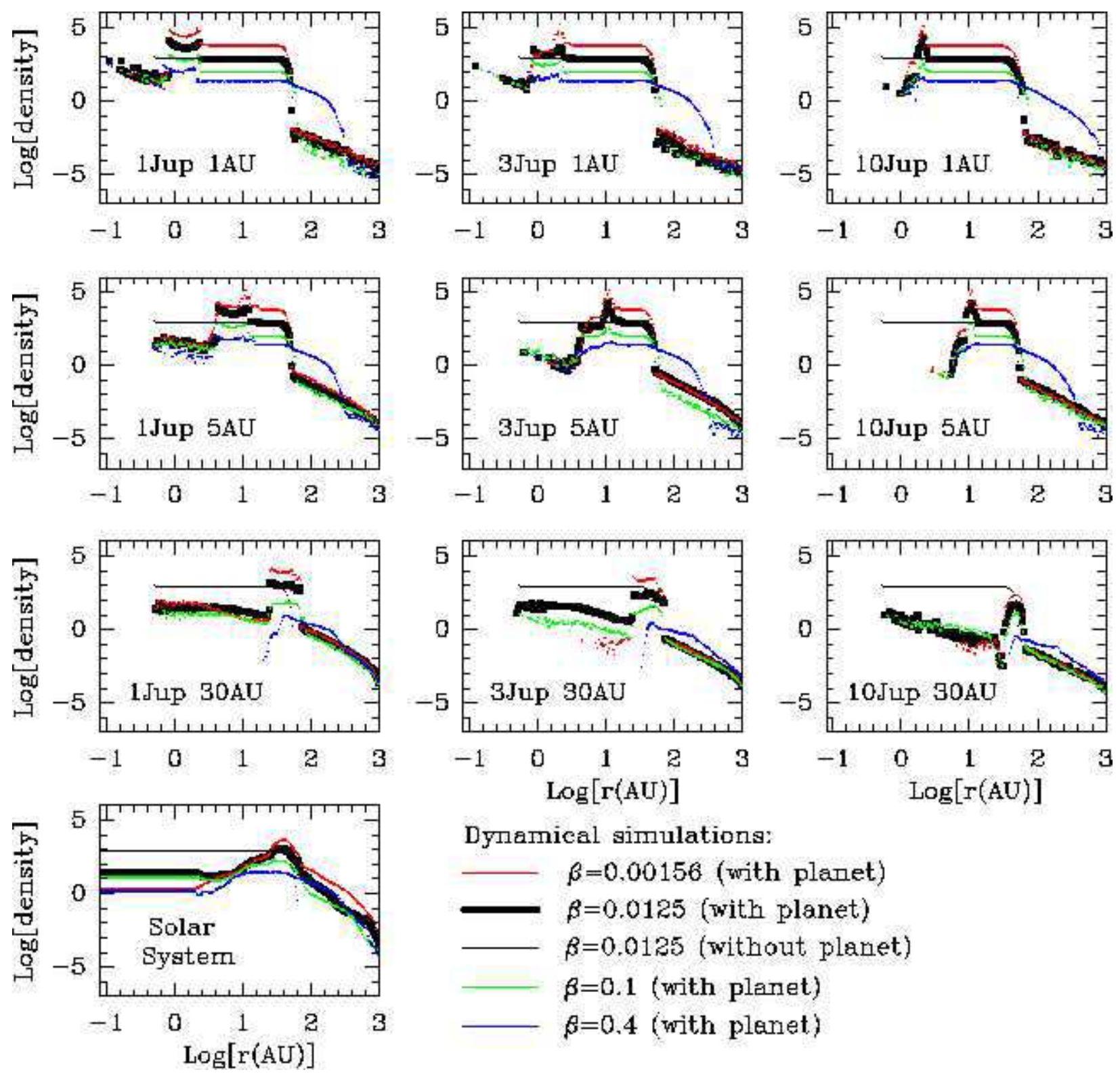

Dynamical simulations:

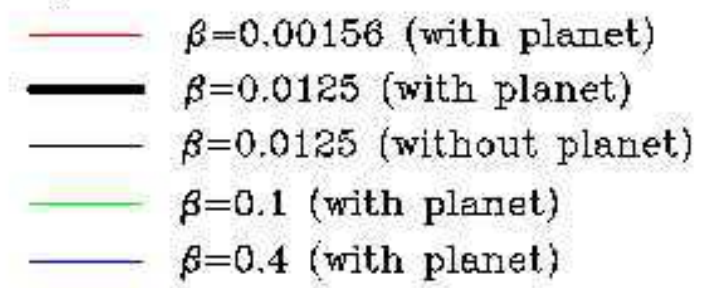

Fig. 2.- Surface density distributions of dust particles with four different $\beta$ values, for different planetary systems (indicated in the individual panels). The units are number of particles per $\mathrm{AU}^{2}$ for a dust production rate of 100 particles per 1000 years (to be later scaled to the correct dust production rate or total disk mass). Large black dots: results from the dynamical simulations when the gravitational perturbation of the planet is taken into account, for dust particles with $\beta=0.0125$. Red: same as before but for $\beta=0.00156 ;$ Green: $\beta=0.1 ;$ Blue: $\beta=0.4$. Small black dots (or thin solid line): results from the dynamical simulations when no planets are present, for dust particles with $\beta=0.0125$. The Solar System model is extrapolated down to $0.01 \mathrm{AU}$ assuming a flat surface density distribution, expected for a collisionless system with grains in circular orbits. 

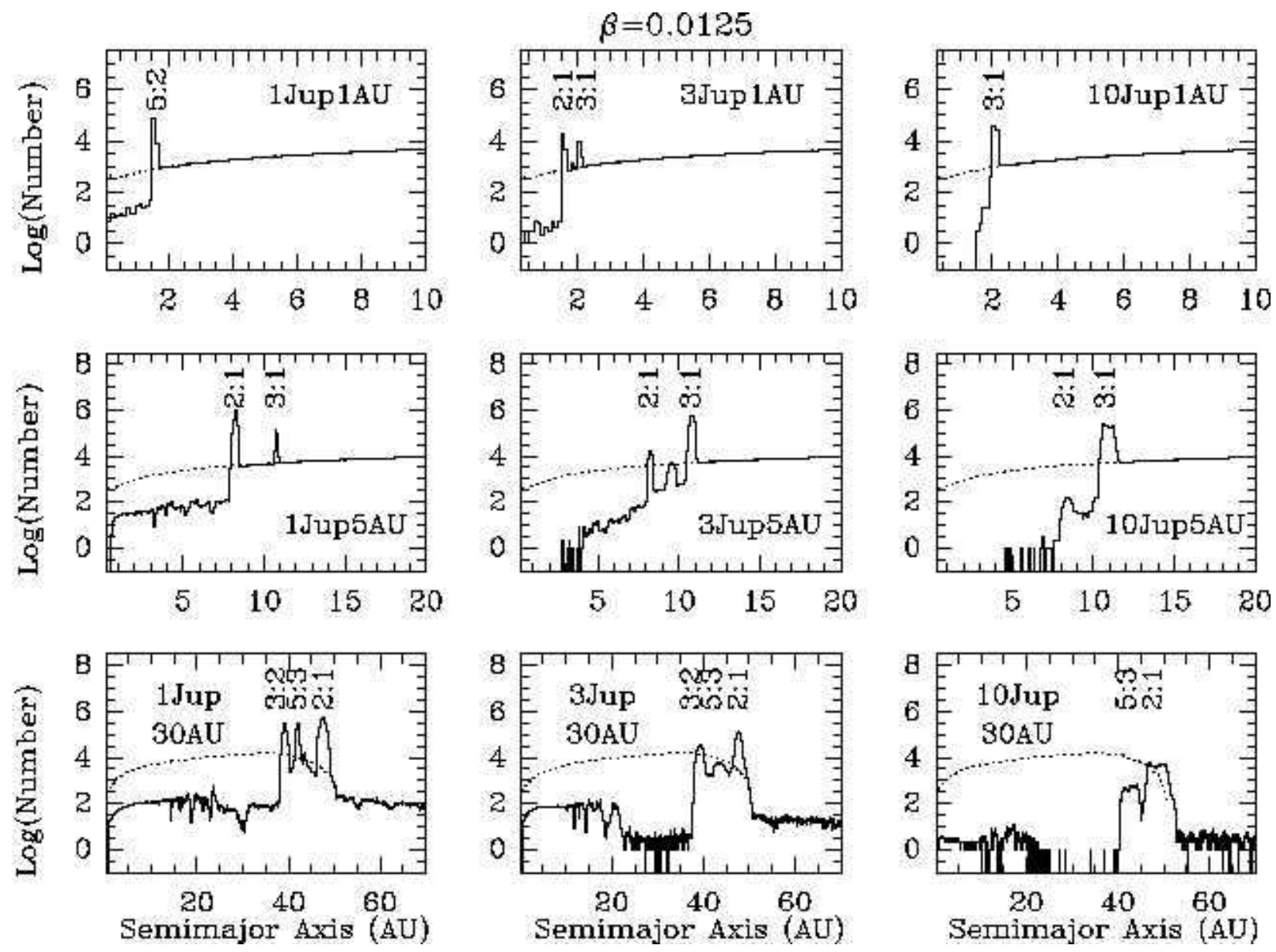

Fig. 3.- "Equilibrium" semimajor axis distributions in logarithmic scale of the dust particles with $\beta=0.0125$, in different single-planet systems (indicated in the individual panels; solid line). The dotted line correspond to a system without planets. The trapping of particles in the exterior MMRs with the planet, and the depletion of particles inside the planet's orbit are the most prominent features in the figure. The y-axis is the number of particles within a given range of semimajor axis. Only the relative numbers are meaningful. 

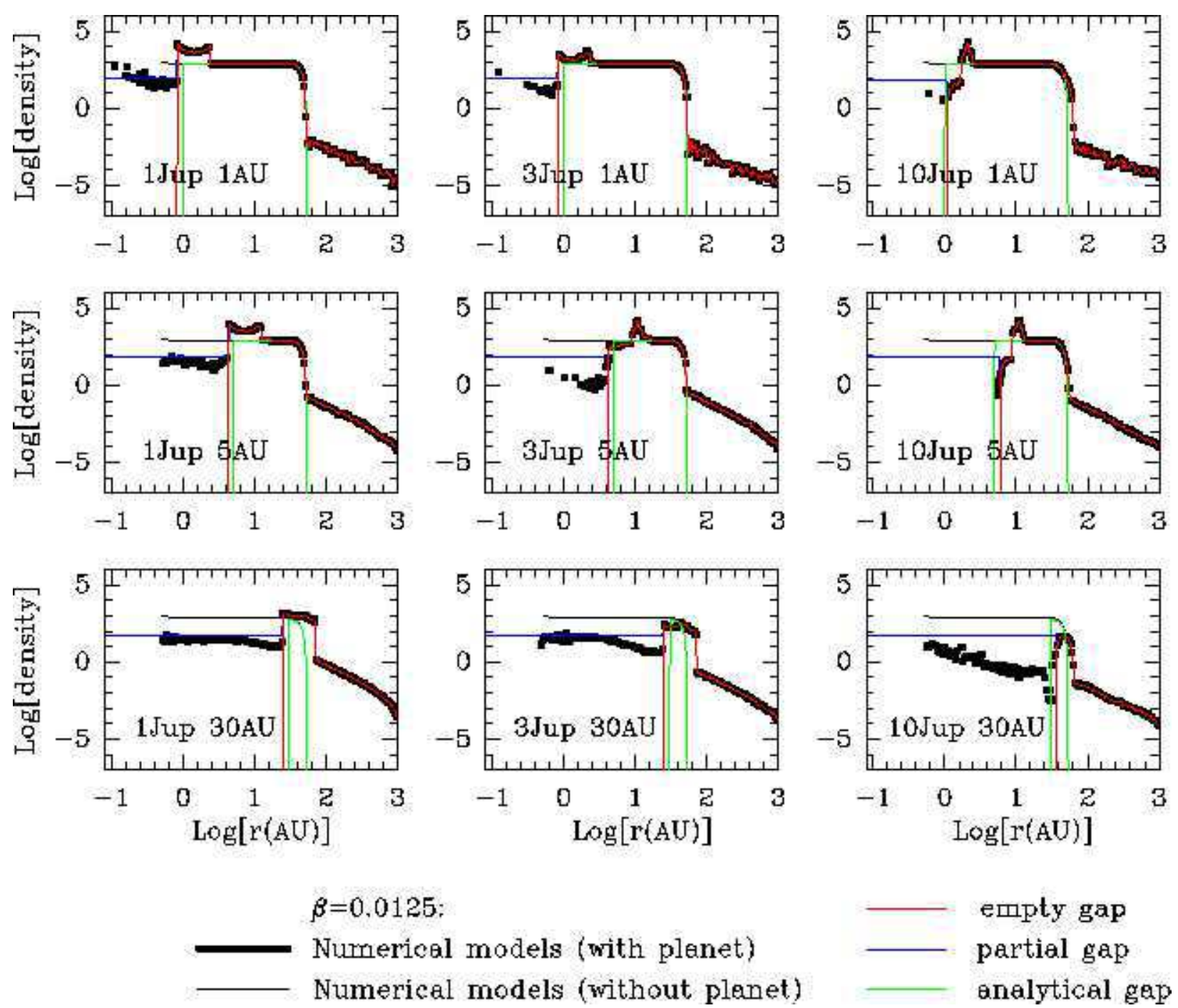

Fig. 4.- Surface density distributions used as input for the radiative transfer code for dust particles with $\beta=0.0125$, for different planetary systems (indicated in the individual panels). Large black dots: results from the dynamical simulations when a planet is present. Small black dots (or thin solid line): results from the dynamical simulations when no planets are present. Red line: surface density distributions with "empty gap". Blue line: surface density distributions with "partial gap". Green line: surface density distributions with "analytical gap". 


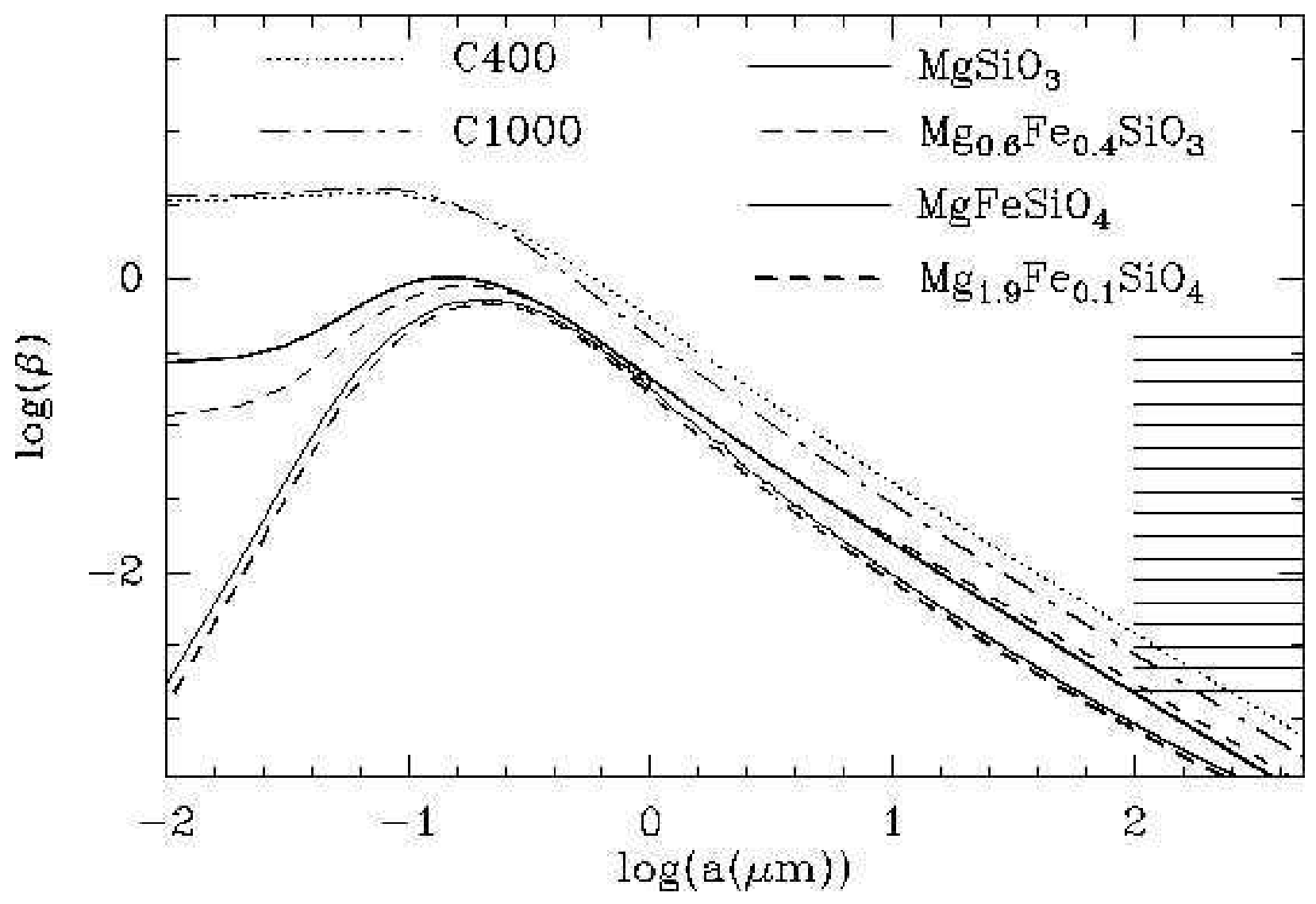

Fig. 5.- Correspondence between $\beta$ and particle radius for the grain chemical compositions under consideration. The horizontal lines at the far right indicate the values of $\beta$ used in the dynamical models. 


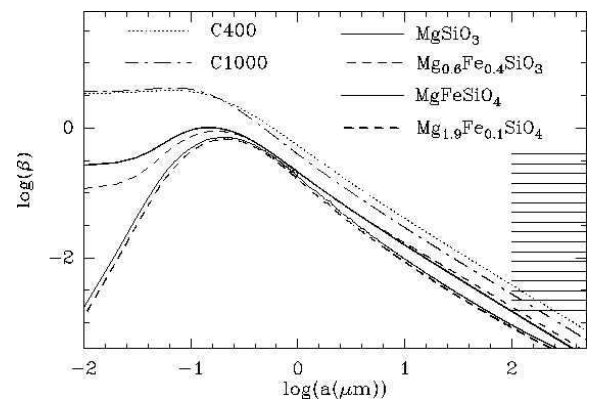

Fig. 6. - SEDs of single particle size disks from the Solar System models. Each panel corresponds to a particular grain chemical composition. Each color corresponds to a different $\beta$ value (or grain size): Red for $\beta=0.4$, Green for $\beta=0.025$ and Blue for $\beta=0.00156$. Solid line: system with 7 planets; Dotted line: system without planets. In all cases the disk is assumed to be at distance of $50 \mathrm{pc}$ and has a mass of $10^{-10} \mathrm{M}_{\odot}$. The squares indicate Spitzer $5-\sigma$ detection limits. 

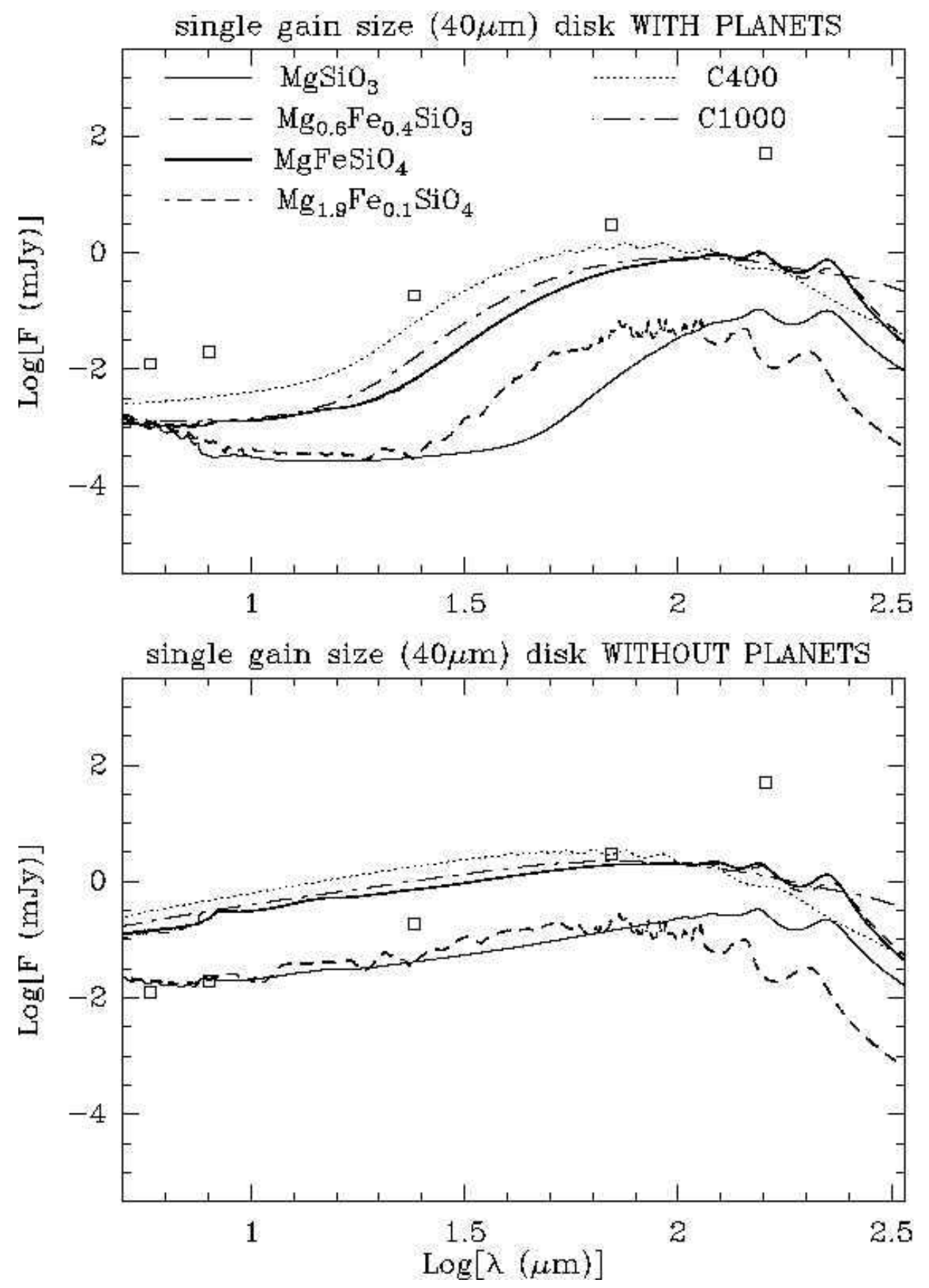

Fig. 7.- SEDs of disks composed of $1 \mu \mathrm{m}$ and $40 \mu \mathrm{m}$ grains, from the Solar System models, and with different grain chemical compositions. 


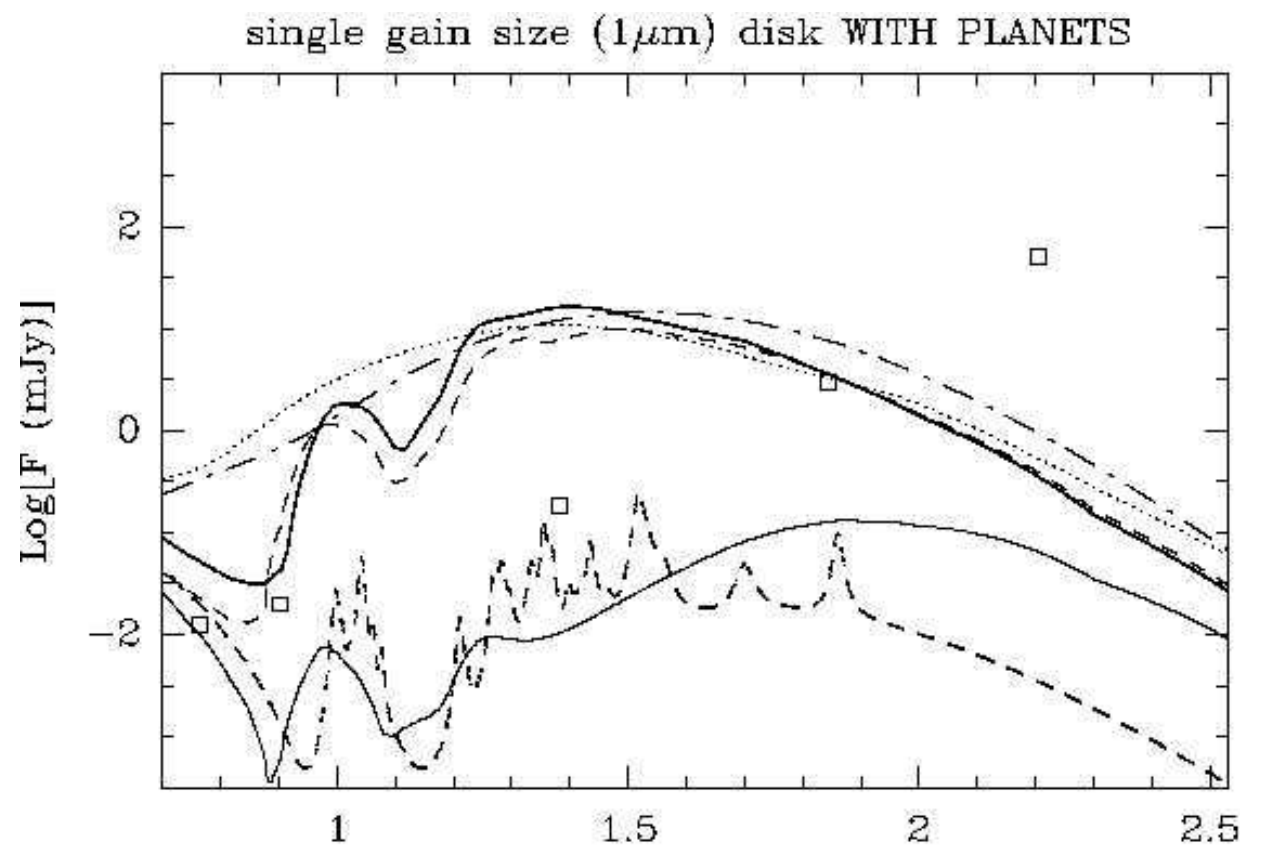

single gain size $(1 \mu \mathrm{m})$ disk WTHHOUT PLANETS

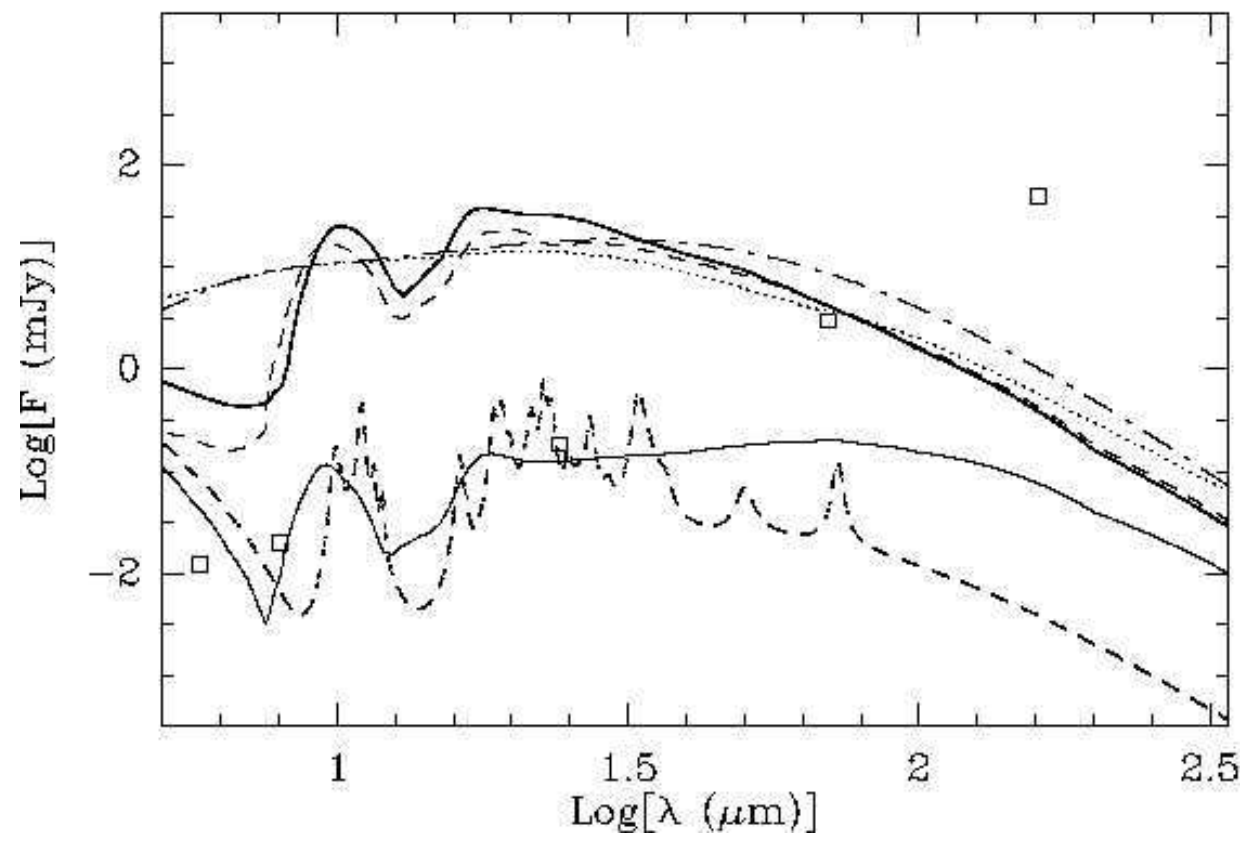



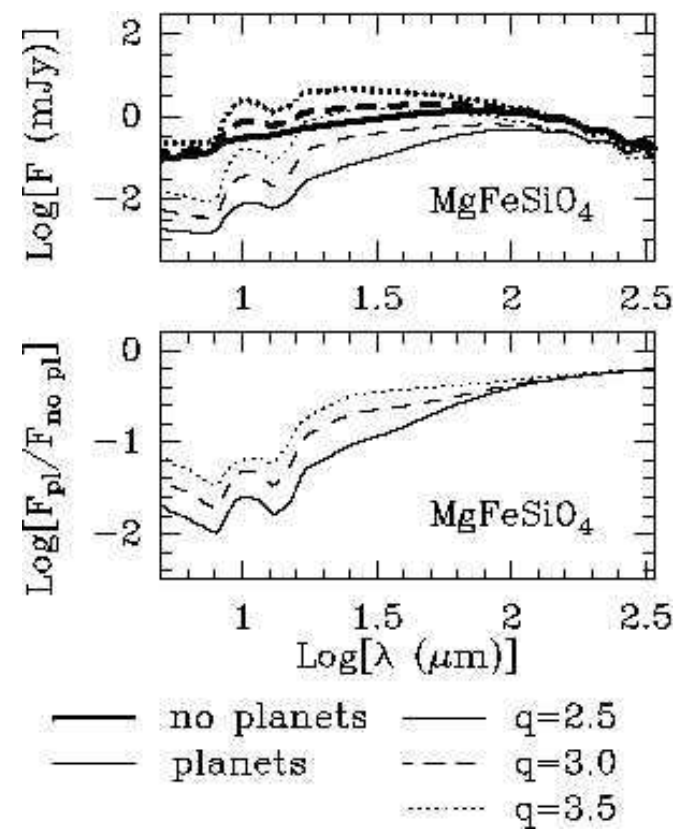

Fig. 8.- (Top panel) SEDs of dust disks composed of $\mathrm{MgFeSiO}_{4}$ grains, for three particle size distribution: $n(b) d b=n_{0} b^{-q}$, with $q=2.5$ (solid line), 3.0 (dashed line) and 3.5 (dotted line). Thick line: system without planets; Thin line: system with Solar System-like planets. The system is at a distance of $50 \mathrm{pc}$ and has a total disk mass of $10^{-10} \mathrm{M}_{\odot}$. (Bottom panel) Ratio of the composed SED arising from a system with planets to that of a system with no planets. 

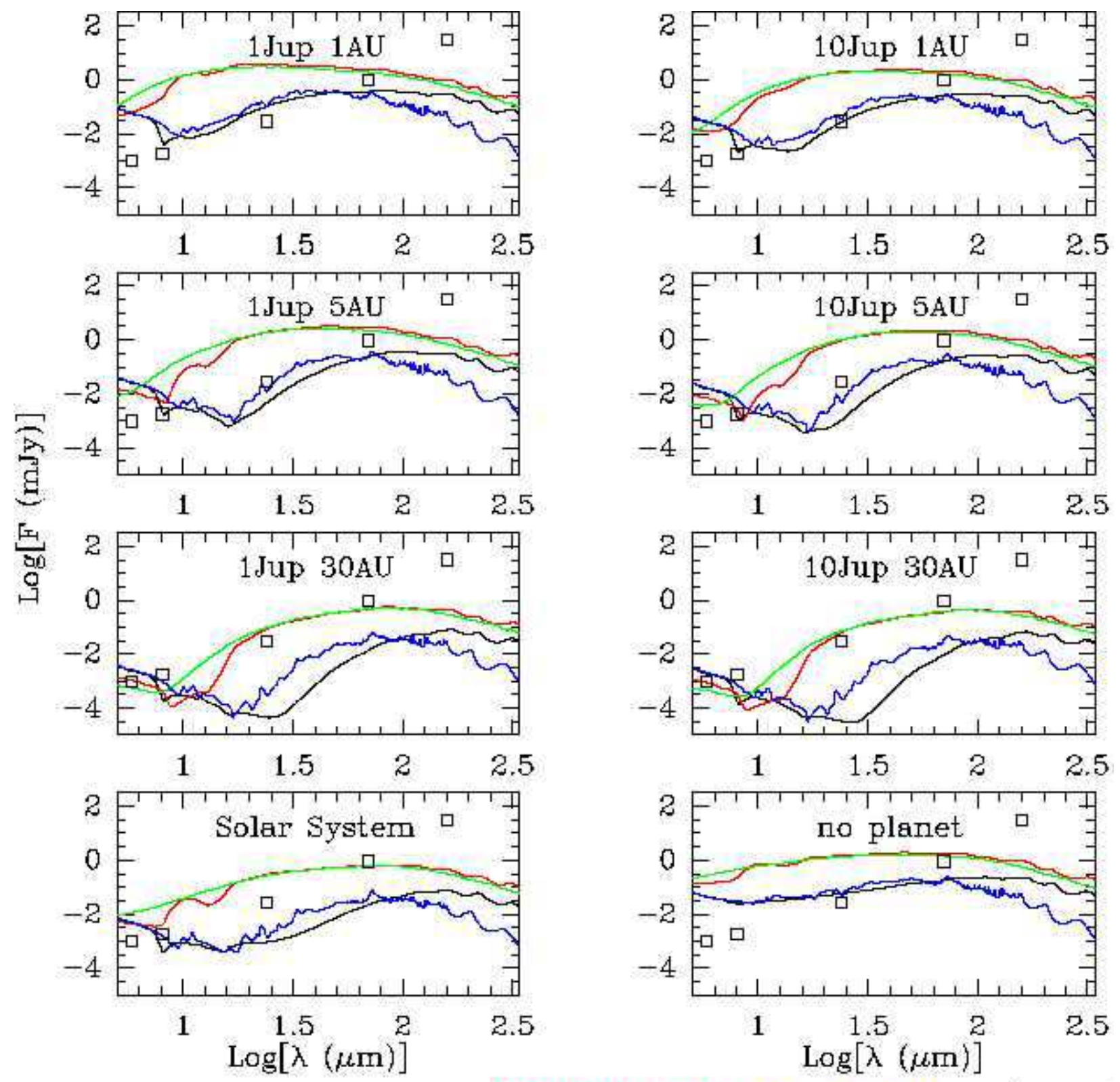

Grain compositions: $\mathrm{MgSiO}_{\mathrm{g}} \quad \mathrm{MgFeSiO}_{4} \mathrm{C} 400 \quad \mathrm{Mg}_{1.9} \mathrm{Fe}_{0.1} \mathrm{SiO}_{4} \quad(\mathrm{q}=3.0)$

Fig. 9.- SEDs of dust disks in the presence of different planetary configurations (indicated at the top of each panel), for four grain chemical compositions (in different colors), and particle size distribution given by $n(b) d b=n_{0} b^{-q}$ with $q=3.0$. In all cases the system is at a distance of $50 \mathrm{pc}$ and has a total disk mass of $10^{-10} \mathrm{M}_{\odot}$. The squares indicate Spitzer 5- $\sigma$ detection limits. 

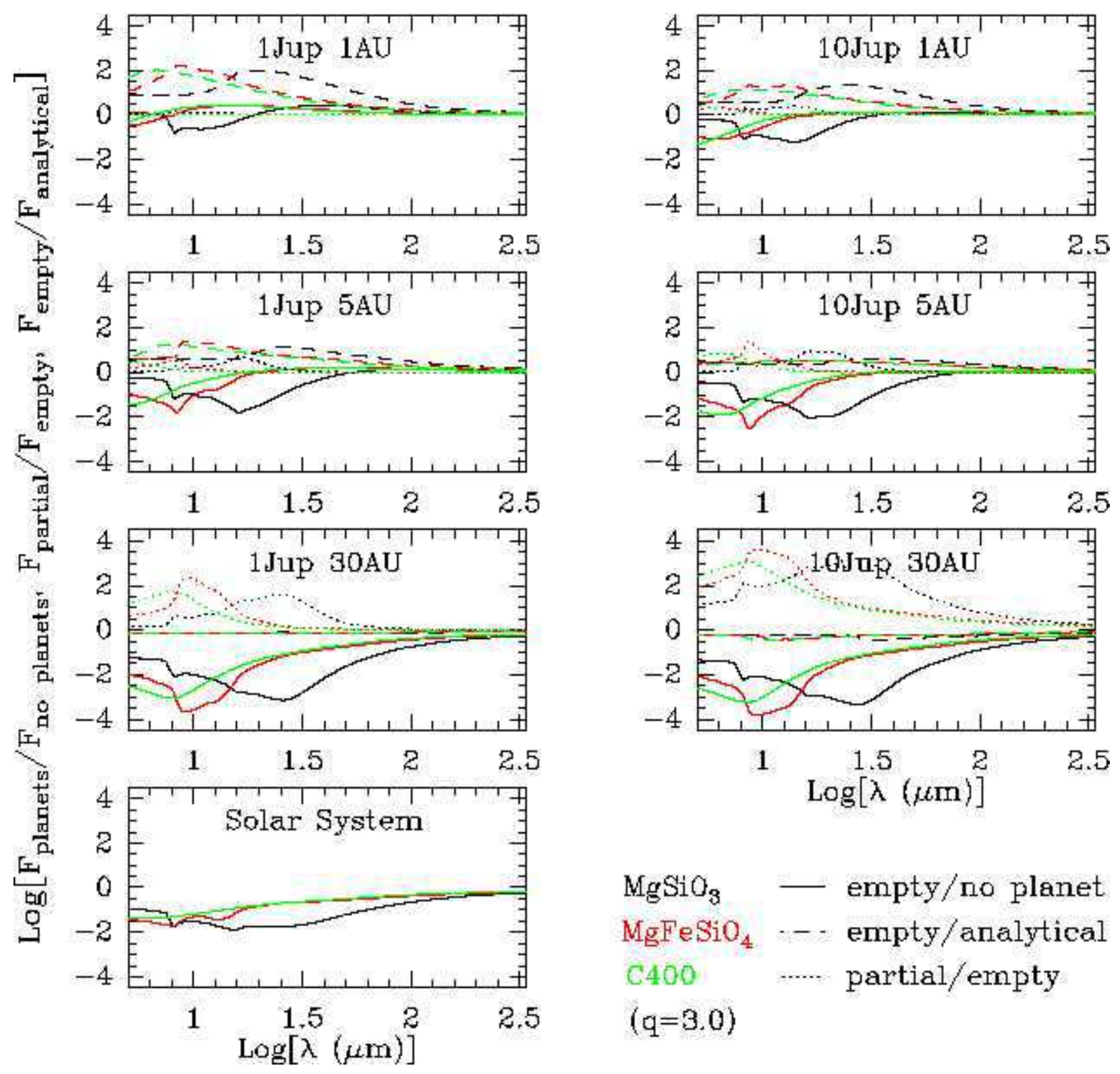

\section{$\mathrm{MgSiO}_{3} \quad$ empty/no planet} $\mathrm{MgFeSiO}_{4}$-.. empty/analytical $\mathrm{C} 400 \quad \cdots .$. partial/empty $(q=3.0)$

Fig. 10.- Ratio of the SEDs that arise from different modeled disks: Solid line: $\mathrm{F}_{\text {planet }} / \mathrm{F}_{\text {no planet }}\left(=\mathrm{F}_{\text {empty gap }} / \mathrm{F}_{\text {no planet }}\right) ;$ Dotted line: $\mathrm{F}_{\text {partial gap }} / \mathrm{F}_{\text {empty gap }} ;$ and Dashed line: $\mathrm{F}_{\text {empty gap }} / \mathrm{F}_{\text {analytical gap. }}$ The different colors correspond to different grain chemical compositions. In all cases $q=3.0$. 

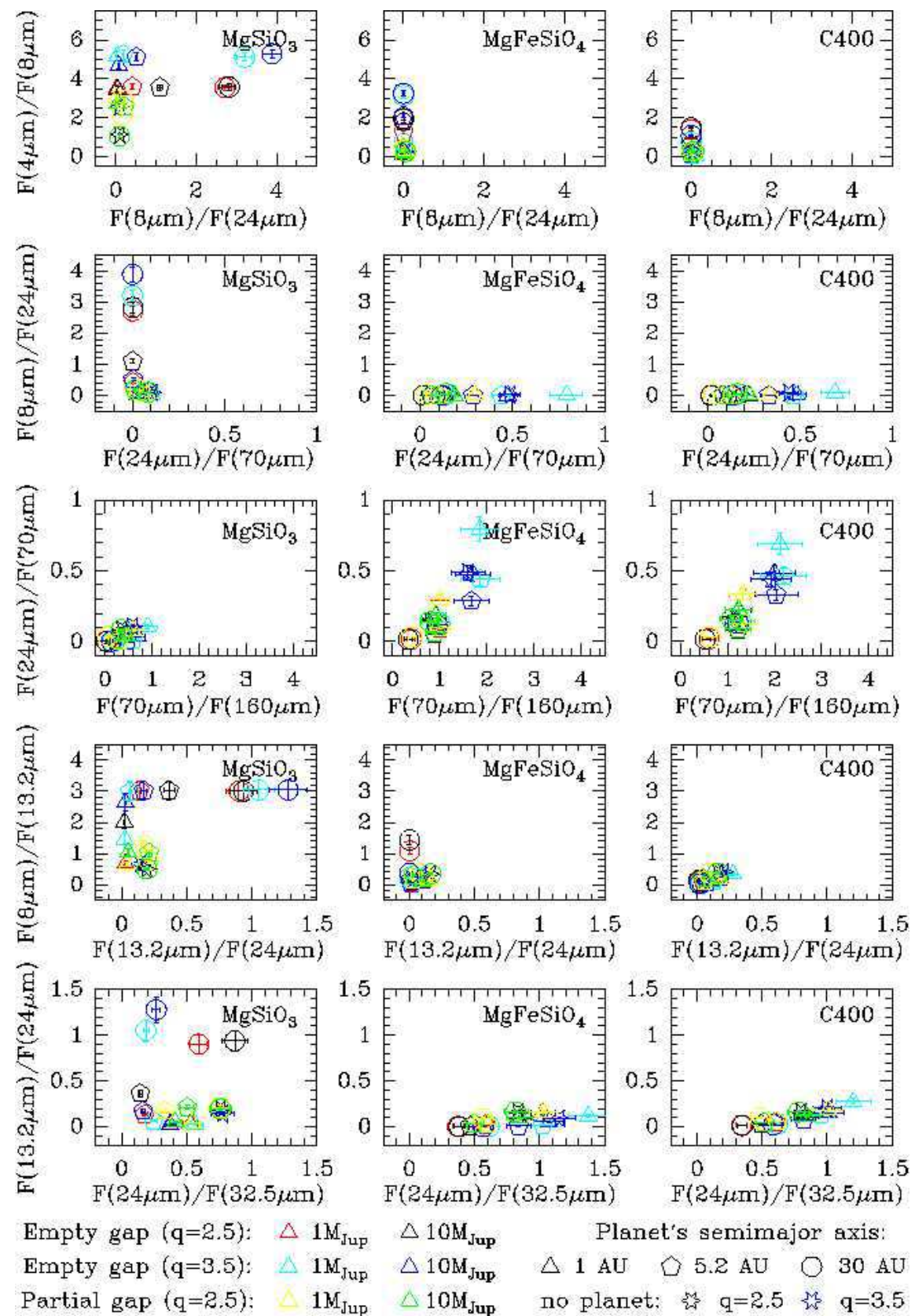

Fig. 11. - Predicted Spitzer broadband colors for the SEDs in Figure 9. The different symbols correspond to different planetary systems. The symbol shape indicates the planet semimajor axis. The symbol color indicates planet mass, power law index for the particle size distribution, and whether the gap is empty or partially filled. The error bars indicate (optimistic) Spitzer calibration uncertainties: $3 \%$ for IRAC in all bands; $10 \%$ for IRS; $5 \%$ for MIPS $24 \mu \mathrm{m} ; 10 \%$ for MIPS $70 \mu \mathrm{m}$; $20 \%$ for MIPS $160 \mu \mathrm{m}$. 


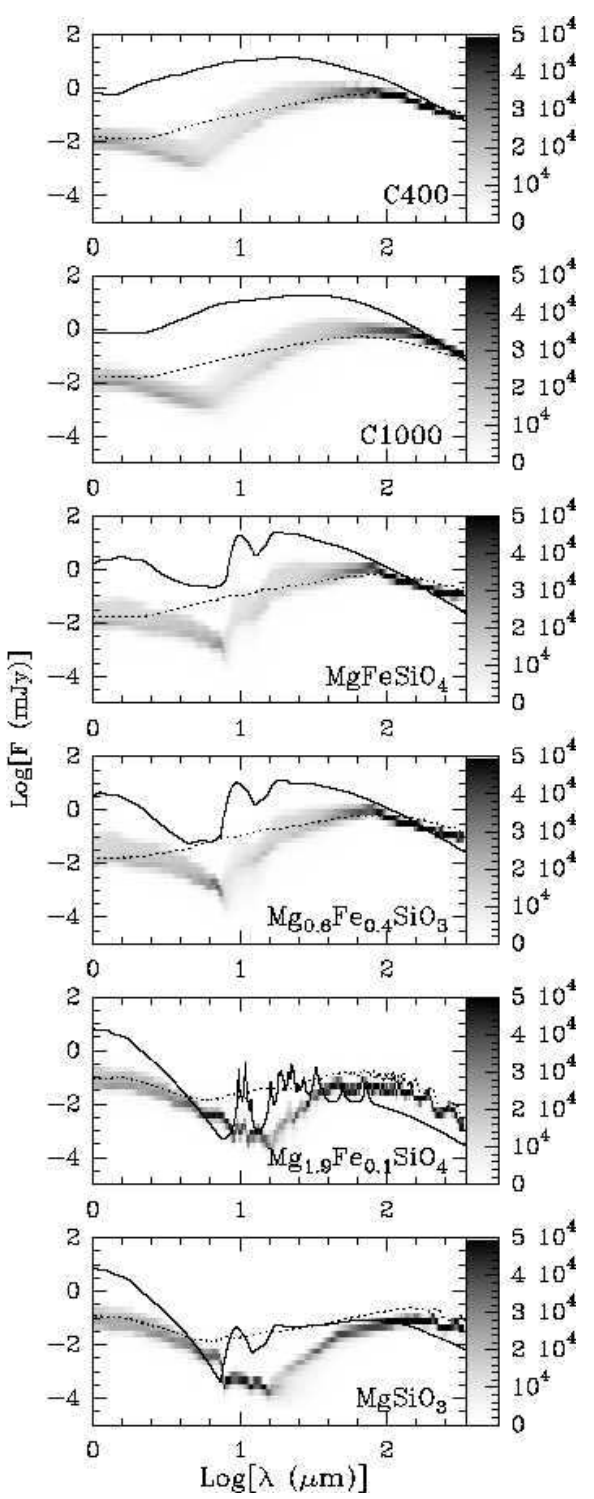

Fig. 12. - SED probability distributions. The gray - scale corresponds to SEDs of disks with embedded planets between $1 \mathrm{AU}$ and $30 \mathrm{AU}$, and with different dust depletion factors. The solid and dotted lines represent the SEDs from the disks without planets for $\beta$-values of 0.4 and 0.00156 , respectively. Each panel corresponds to a different grain mineralogy. 


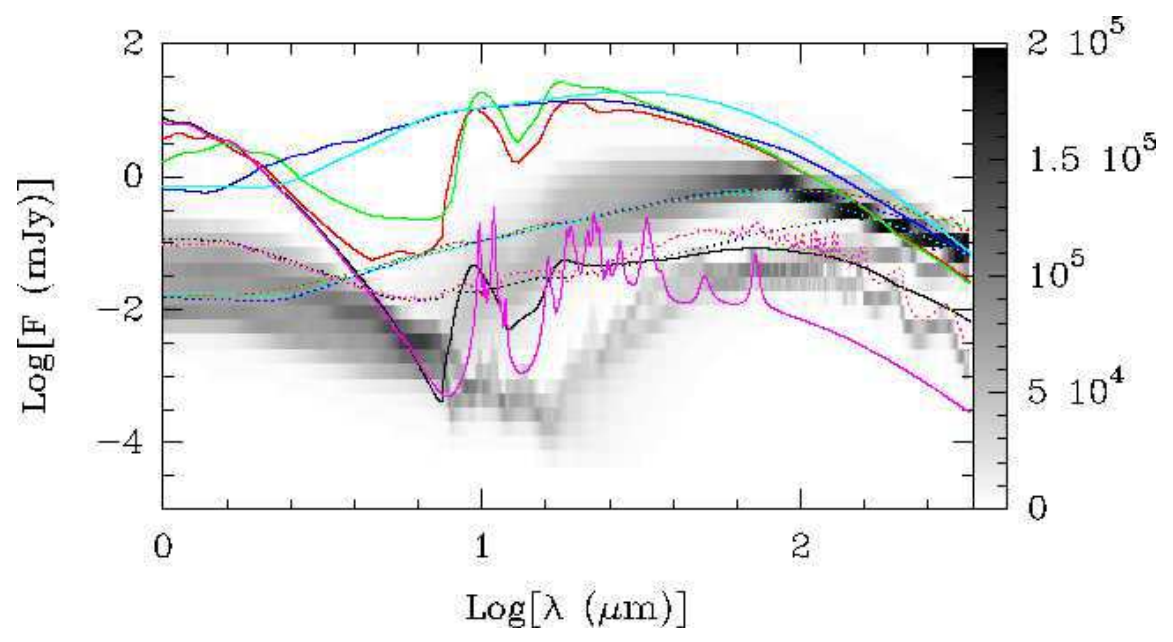

Fig. 13.- Same as Figure 12, with the six mineralogies all plotted together in gray-scale (a total of $21 \times 36 \times 11 \times 6=49896$ SEDs). The SEDs from the disks without planets are shown in the solid and dotted lines, with each color corresponding to one mineralogy. black: $\mathrm{MgSiO}_{3} ;$ red: $\mathrm{Mg}_{0.6} \mathrm{Fe}_{0.4} \mathrm{SiO}_{3}$; green: $\mathrm{MgFeSiO}_{4} ;$ magenta: $\mathrm{Mg}_{1.9} \mathrm{Fe}_{0.1} \mathrm{SiO}_{4}$; dark blue: $\mathrm{C} 400$; light blue: $\mathrm{C} 1000$. 

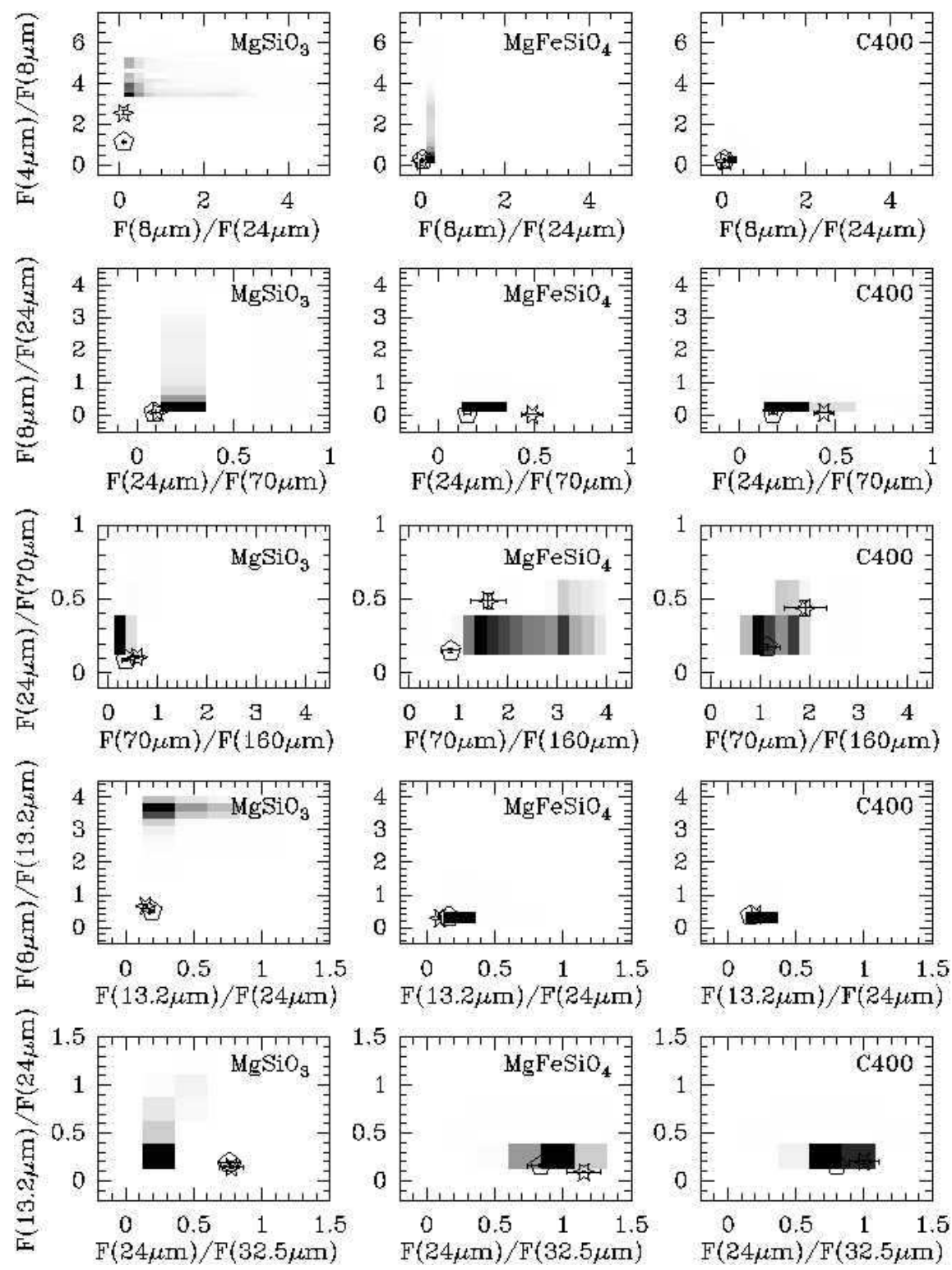

Fig. 14.- Predicted Spitzer broadband colors for the SEDs in Figure 12. The gray - scale corresponds to the colors from disks with embedded planets between $1 \mathrm{AU}$ and $30 \mathrm{AU}$, and with different dust depletion factors. The symbols (pentagon and star) correspond to the colors derived from disks without planets (for power law indexes of 2.5 and 3.5, respectively). The error bars indicate (optimistic) Spitzer calibration uncertainties: $3 \%$ for IRAC in all bands; $10 \%$ for IRS; $5 \%$ for MIPS $24 \mu \mathrm{m}$; 10\% for MIPS $70 \mu \mathrm{m} ; 20 \%$ for MIPS $160 \mu \mathrm{m}$. 

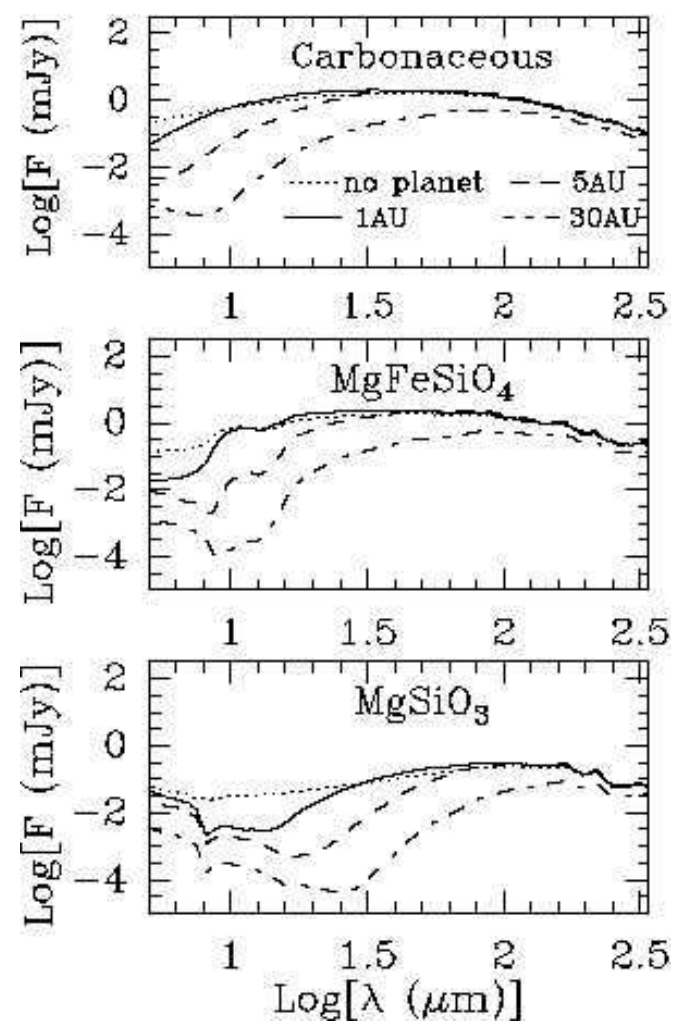

Fig. 15. - SEDs of dust disks in the presence of different planetary configurations [solid: $3 \mathrm{M}_{J u p}$ at $1 \mathrm{AU}$; dashed: $3 \mathrm{M}_{\text {Jup }}$ at $5 \mathrm{AU}$; dashed - dotted: $3 \mathrm{M}_{\text {Jup }}$ at $30 \mathrm{AU}$; dotted: system without planets]. Results are shown for three grain chemical compositions (indicated in each panel), and particle size distribution given by $n(b) d b=n_{0} b^{-q}$ with $q=3.0$. In all cases the system is at a distance of $50 \mathrm{pc}$ and has a total disk mass of $10^{-10} \mathrm{M} \odot$. 

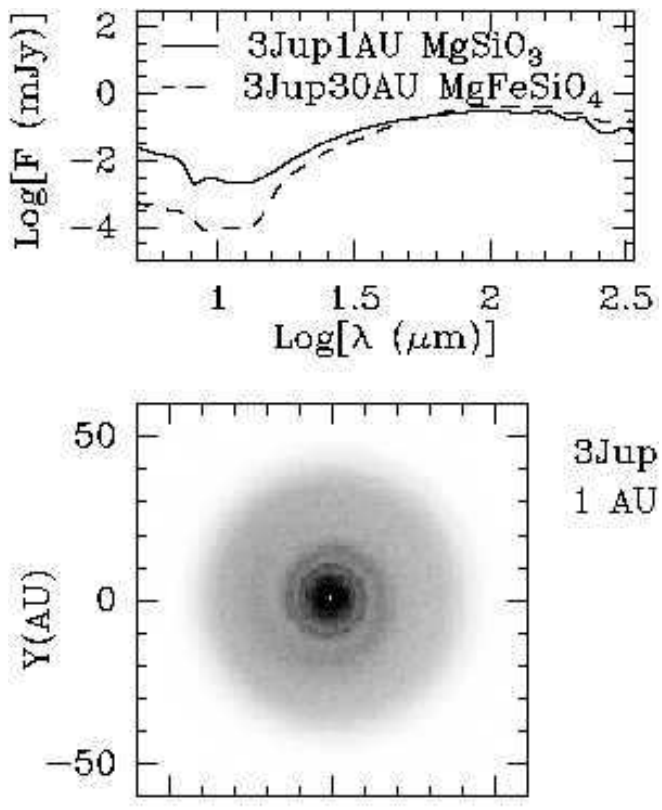

BJup

$1 \mathrm{AU}$

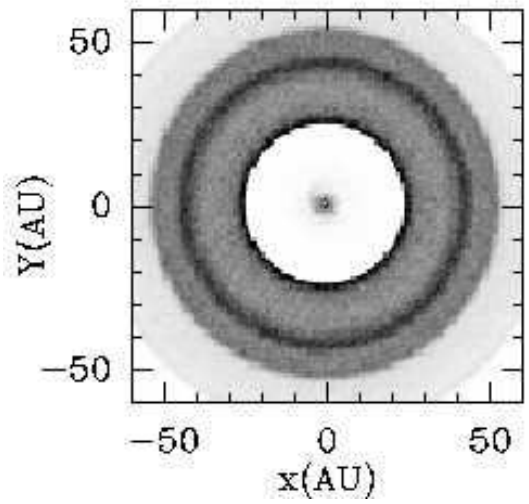

3Jup $30 \mathrm{AU}$

Fig. 16.- (top) Possible degeneracy between the grain chemical composition and the location of the planet clearing the gap. Solid line: SED of dust disk composed of $\mathrm{MgSiO}_{3}$ grains with a $3 \mathrm{M}_{\text {Jup }}$ planet at $1 \mathrm{AU}$; dashed line: same for $\mathrm{MgFeSiO}_{4}$ grains with a $3 \mathrm{M}_{\text {Jup }}$ planet at $30 \mathrm{AU}$. In both cases $q=2.5$. (middle) and (bottom) Brightness density distributions at $70 \mu \mathrm{m}$ (assuming graybody emission from $12 \mu \mathrm{m}$ grains) expected from a disk with a $3 \mathrm{M}_{J u p}$ planet at $1 \mathrm{AU}$ and 30 AU, respectively (shown in arbitrary units). High resolution images are needed to solve the degeneracy. 